\title{
Total cross-section for Higgs boson hadroproduction with anomalous Standard-Model interactions
}

\author{
Journal Article \\ Author(s): \\ Anastasiou, Charalampos; Buehler, Stephan; Herzog, Franz; Lazopoulos, Achilleas \\ Publication date: \\ 2011-12 \\ Permanent link: \\ https://doi.org/10.3929/ethz-b-000046198 \\ Rights / license: \\ Creative Commons Attribution 4.0 International \\ Originally published in: \\ Journal of High Energy Physics 2011(12), https://doi.org/10.1007/JHEP12(2011)058
}




\title{
Total cross-section for Higgs boson hadroproduction with anomalous Standard-Model interactions
}

\author{
Charalampos Anastasiou, Stephan Buehler, Franz Herzog and Achilleas Lazopoulos \\ Institute for Theoretical Physics, ETH Zurich, \\ 8093 Zurich, Switzerland \\ E-mail: babis@phys.ethz.ch, buehler@itp.phys.ethz.ch, \\ fherzog@itp.phys.ethz.ch, lazopoli@itp.phys.ethz.ch
}

ABSTRACT: We present a new program (iHixs) which computes the inclusive Higgs boson cross-section at hadron colliders. It incorporates QCD corrections through NNLO, real and virtual electroweak corrections, mixed QCD-electroweak corrections, quark-mass effects through NLO in QCD, and finite width effects for the Higgs boson and heavy quarks. iHixs can be used to obtain the most precise cross-section values in fixed order perturbation theory in the Standard Model. In addition, it allows for a consistent evaluation of the cross-section in modified Higgs boson sectors with anomalous Yukawa and electroweak interactions as required in extensions of the Standard Model. iHixs is interfaced with the LHAPDF library and can be used with all available NNLO sets of parton distribution functions.

Keywords: Higgs Physics, NLO Computations, Beyond Standard Model, Standard Model ARXIV EPRINT: 1107.0683 


\section{Contents}

1 Introduction 1

2 Features of iHixs $\quad 2$

2.1 Components of the gluon fusion cross-section in iHixs 3

2.2 Components of the bottom-quark fusion cross-section in iHixs 4

3 Higgs boson interactions $\quad 5$

4 Hadronic and partonic cross sections 5

5 The gluon fusion process through NLO QCD 9

5.1 LO: $g g \rightarrow h \quad 9$

$\begin{array}{lll}5.2 & \text { NLO: } g g \rightarrow h+g & 10\end{array}$

$\begin{array}{lll}5.3 & \text { NLO: } q \bar{q} \rightarrow h+X & 11\end{array}$

$5.4 \quad$ NLO: $q g \rightarrow h+X \quad 11$

5.5 NLO: mixed QCD-EW corrections to $q \bar{q} \rightarrow H+g$ and $q g \rightarrow h+g \quad 11$

6 Beyond the NLO QCD

6.1 The Standard Model Wilson coefficient with anomalous Yukawa and elec$\begin{array}{ll}\text { troweak couplings } & 14\end{array}$

$\begin{array}{lll}\text { 6.2 Improving on the effective theory approximation } & 15\end{array}$

$\begin{array}{llr}7 & \text { Numerical results in gluon fusion } & \mathbf{1 6}\end{array}$

$\begin{array}{lll}7.1 & \text { Perturbative convergence and scale uncertainty } & 16\end{array}$

$\begin{array}{lll}7.2 & \text { PDF comparison } & 17\end{array}$

$\begin{array}{lll}7.3 \text { Top quark width } & 18\end{array}$

$\begin{array}{lll}7.4 & \text { Finite Higgs boson width effects } & 18\end{array}$

7.5 Inclusive Higgs boson production in the presence of a fourth generation of quarks 23

8 The Higgs cross-section for a variable bottom-quark Yukawa interaction 24

9 The iHixs program $\quad 28$

$\begin{array}{lll}9.1 \text { Usage } & 28\end{array}$

$\begin{array}{lll}9.2 & \text { Setting options and variables } & 29\end{array}$

$\begin{array}{lll}9.3 & \text { Libraries used } & 31\end{array}$

10 Conclusions $\quad 31$

A Tables of Higgs cross-sections 
B.1 Master integral definitions 36

B.2 $A_{\text {gggH }} \quad 36$

$\begin{array}{lll}\text { B.3 } & A_{q \bar{q} g H} & 38\end{array}$

B.4 $A_{\text {ewk }}$

$\begin{array}{lll}\text { B.5 } A_{\mathrm{ewk}}^{m_{t}} & 39\end{array}$

\section{Introduction}

A major objective of experimental high energy physics is the discovery of the Higgs boson. Production cross-sections at hadron accelerator experiments are expected to be small. However, they are sufficiently significant in order for the Standard Model (SM) Higgs boson to be discovered soon in a variety of signatures. A remarkable progress in this direction has been made after the direct searches of LEP [1]. The TEVATRON [2] experiments have already demonstrated sensitivity to Higgs boson cross-sections of magnitudes as in the Standard Model. ATLAS and CMS have published limits on the inclusive cross-section using first LHC data [3, 4] accumulated in 2010. These searches are expected to yield tight constraints in their forthcoming updates with data from the 2011 runs.

Interactions in the Higgs sector of the Standard Model are only indirectly constrained experimentally. Theory-wise, a modified Higgs sector is a prerequisite for extensions of the Standard Model with a more appealing UV completion. When computing Higgs boson production cross-sections, it is necessary to allow for the possibility of Higgs boson interactions with modified couplings from their Standard-Model values.

At a hadron collider, the inclusive Higgs boson cross-section is most sensitive to the values of the quark Yukawa couplings. The main hadroproduction mechanism is gluon fusion which receives sizable contributions from top and bottom-quark loops. In Standard Model extensions, the mass of elementary particles may not originate entirely from the Higgs mechanism and, in addition, not yet discovered quarks may be postulated which also contribute to the cross-section. Very enhanced or very suppressed Higgs boson crosssections may be obtained, as it occurs for example in a Standard Model with a fourth generation [5-8] and composite Higgs boson models [9-11] correspondingly.

Direct production from quark partons in hadrons (mainly the bottom and charm) may also become sizable in models with more than one Higgs doublet [12] or dynamically generated Yukawa couplings $[13,14]$. In models with a perturbative Higgs sector, gluon fusion is likely less sensitive to modifications of the Higgs boson couplings to electroweak gauge bosons; these start contributing to the production of a single Higgs boson only at the two-loop level. The bulk of the generally small electroweak corrections is due to loops with light Standard Model quarks which have very well constrained electroweak couplings.

A light Higgs boson is expected to have a small decay width in the Standard Model. The width could be different in extensions of the Standard Model. For example, the Higgs boson could have a significant invisible decay width in scenaria with hidden sectors [15-17]. The width of the Higgs boson is expected to be large also for scenaria where a heavy mass is not disallowed. 
The objective of this publication is to provide precise theory predictions for the production cross-section of a Higgs boson in the Standard Model and extensions which alter the interactions of the Higgs boson and quarks or electroweak gauge bosons. This publication is accompanied with a computer program, iHixs, for the precise calculation of inclusive Higgs boson cross-sections at the Tevatron and the LHC in such a general setup.

\section{Features of iHixs}

In the last two decades many theoretical studies lead to improved estimates of the Standard Model Higgs boson total cross-section at hadron colliders with a level of 10-20\% precision. Using, extending and combining the available theoretical calculations has been proven to be a non-trivial task for both theorists and experimentalists. iHixs is an easy to use program which produces accurate predictions for the Higgs cross-section in the Standard Model and can be adapted readily for a large class of extensions of the Standard Model.

For the numerical evaluation of loop and phase-space integrals we have used a new Fortran package [18] of harmonic polylogarithms with complex arguments. For one-loop box and triangle master integrals with different internal masses we have used the library OneLOop of ref. [19, 20] which allows for complex masses. We have tested our implementation of all other one-loop master integrals against both OneLOop and the numerical program of ref. [21].

iHixs computes the inclusive Higgs boson cross-section through next-to-next-toleading-order (NNLO) in perturbative QCD. A large source of uncertainty for Higgs boson cross-sections at hadron colliders is the precision in the determination of the parton densities. It is therefore important to compare the effect of diverse existing determinations of parton densities on the Higgs cross-section, as well as future sets which will incorporate refined measurements and theory. iHixs allows these studies effortlessly. It is interfaced through the LHAPDF library [22] with all available parton distribution functions with a consistent evolution at NNLO [23-25]. Other sets of the library can be employed by simple modifications of the code.

iHixs allows the study of the Higgs boson invariant mass distribution for a finite width of the Higgs boson and compute the cross-section sampling over a Breit-Wigner distribution. This assumes that matrix-elements for the production of a Higgs boson decay factorize in matrix-elements for the production of a Higgs boson times matrix-elements for the decay. A grid of values for the branching rations and the decay width of the Higgs boson in the mass range of interest is a necessary ingredient for iHixs. For the Standard Model (or models with very similar Higgs decay rates), iHixs uses a grid of values for the width and branching ratios that we produced with the program HDECAY version 3.532 [26]. To study models where the width and branching ratios differ significantly from the SM, the user has to provide his own grid, in the form of a simple text file.

iHixs includes perturbative contributions for two production processes:

- gluon fusion

- bottom-quark fusion 


\subsection{Components of the gluon fusion cross-section in iHixs}

The cross-section for the gluon fusion process in iHixs comprises:

1. Leading order and next-to-leading order (NLO) QCD effects with exact quark-mass dependence. The number of quarks and their Yukawa couplings are arbitrary.

The required two-loop amplitude has been first computed in ref. [27, 28] where it was presented in the form of an integral representation. ${ }^{1}$ This was later expressed in terms of harmonic polylogarithms with the method of series expansion and resummation in ref. [31]. Independent analytic evaluations were performed in refs. [32, 33].

The real radiation matrix-elements have been computed in ref. [34, 35] and recomputed for the purposes of several other publications including this one. Numerical implementations of the NLO QCD cross-section with full quark-mass effects in the Standard Model were made in ref. [28] and in refs. [36, 37].

2. NNLO QCD corrections, using heavy quark effective theory (HQET).

The NNLO Wilson coefficient for an arbitrary number of heavy quarks and Yukawa couplings has been computed in ref. [11]. In the special case of Standard-Model Yukawa couplings this is equivalent to the Wilson coefficient of ref. [38], while in the case of integrating out only a single heavy quark it yields the Wilson coefficient in refs. $[39,40]$.

The NNLO phase-space integrated matrix-elements in HQET have been first evaluated in their threshold limit $[41,42]$. The complete NNLO correction has been computed in refs. [43-45].

3. Two-loop electroweak corrections at leading order in $\alpha$.

These include the full Standard Model contributions to the amplitude as computed in ref. $[46,47] .{ }^{2}$ For a light Higgs boson, a dominant contribution to the two-loop amplitude is due to loops with light quarks [48]. We allow for a common re-scale factor of the $H W W$ and $H Z Z$ couplings. This should be a sufficient parameter for models which have a custodial symmetry protection in order to comply with stringent constraints from electroweak precision tests. ${ }^{3}$

4. One-loop electroweak corrections for the real radiation processes $q \bar{q} \rightarrow g h$ and $q g \rightarrow q h$.

This amplitude has been first computed in ref. [49]. In this paper, we perform an independent calculation and present analytic formulae in terms of a basis of finite

\footnotetext{
${ }^{1}$ First calculations of the two-loop amplitude in the infinite top-quark mass limit were performed in refs. $[29,30]$.

${ }^{2}$ We thank the authors of refs. [46, 47] for kindly providing a text file with the numerical values of the two-loop amplitude.

${ }^{3}$ Custodial symmetry protects from large corrections to the $\hat{T}$ parameter; see, for example, the constraints on custodial symmetry breaking operators of the strongly interacting Higgs boson effective Lagrangian in $[10]$.
} 
master integrals adding also contributions with massive quarks in the loop. Our results agree with the limit of zero Higgs boson mass of ref. [49]. ${ }^{4}$ Subprocesses with bottom quarks in the initial state have also been studied in [80] at a differential level.

5. Mixed QCD and electroweak contributions with light quarks.

This contribution can be estimated by means of an effective field theory and the required Wilson coefficient computed in ref. [50].

6. Arbitrary Wilson coefficient for the $H \operatorname{tr}\left(G_{\mu \nu} G^{\mu \nu}\right)$ operator.

New physics at scales higher than the electroweak scale may introduce modifications to the gluon fusion cross-section which cannot be accounted for by modified Yukawa interactions and rescaling electroweak corrections. In such situations, iHixs allows to introduce corrections to the Wilson coefficient of the HQET theory. Given the non-discovery of new states with Tevatron and first LHC data, it is reasonable to anticipate that the new energy frontier is distant enough from the electroweak scale (higher than the top-quark mass) in order for an effective theory approach to be adequate.

\subsection{Components of the bottom-quark fusion cross-section in iHixs}

While the dominant production mode of the Higgs boson in the Standard Model is gluon fusion via a top-quark loop, a considerable correction of about $5 \%$ arises from bottom-quark loops. Another production channel based on bottom-quark fusion is $g g \rightarrow H b \bar{b}$ [51-53]. In this channel the bottom-quarks are predominantly produced collinear to the gluons. The cross-section at fixed order in perturbation theory suffers from large logarithmic terms. However, these can be resummed into the bottom parton density, leading to another (mostly) single Higgs-boson production mechanism $b \bar{b} \rightarrow H$ [54-56]. We have therefore included this channel into iHixs.

The cross-section for bottom-quark fusion process in iHixs comprises the NNLO crosssection calculation of Harlander and Kilgore in ref. [57]. This cross-section is important when the Yukawa couplings to bottom quarks are enhanced, as it may happen in models with more than one Higgs doublet. We have implemented the analytic formulae of ref. [57] and computed the scale-dependent terms of the cross-section separately. We have checked that our numerical code agrees with the publicly available program of ref. [58].

We included the bottom-fusion process in iHixs for the purposes of facilitating the simultaneous study of enhanced bottom Yukawa couplings in the production of a Higgs boson from bottom-quarks and in gluon fusion via bottom-quark loops. We note that the iHixs program can be adapted easily in order to compute the cross-section for Higgs production via the fusion of lighter quarks, such as the charm-quark, if necessary [14].

\footnotetext{
${ }^{4}$ We were unable to compare with the analytic expressions for the electroweak amplitude of ref. [49] due to the lack of an exact definition of the contributing "finite parts" from the divergent master integrals which were chosen as a basis.
} 


\section{Higgs boson interactions}

We consider a Higgs boson with interactions described by the Feynman rules:
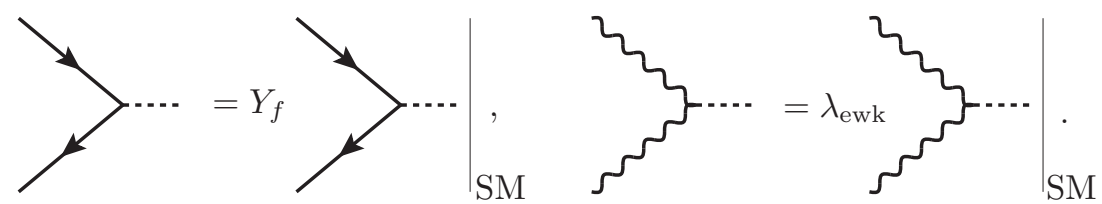

The triple-vertex Higgs-quark-quark is the product of an arbitrary, flavor dependent factor $Y_{f}$ and the analogous Feynman rule in the Standard Model. iHixs permits an arbitrary number of quark flavors $N_{f}$ in order to accommodate extensions of the Standard Model with novel quarks.

The Standard Model Feynman rules for the $H-W-W$ and $H-Z-Z$ vertices are rescaled by a global factor $\lambda_{\text {ewk }}$. We did not find it necessary to introduce a separate re-scaling factor for the $\mathrm{W}$ and $\mathrm{Z}$ boson vertices. The ratio of the coefficients of the corresponding operators is fixed by the custodial symmetry and very tightly constrained by electroweak precision tests [10].

\section{Hadronic and partonic cross sections}

We consider the production of a Higgs boson, $\mathrm{H}\left(p_{H}\right)$, at a hadron collider, which decays to some combination of final state particles that will be collectively denoted by $\left\{H_{\text {final }}\right\}$ :

$$
\operatorname{hadron}_{1}\left(P_{1}\right)+\operatorname{hadron}_{2}\left(P_{2}\right) \rightarrow\left\{\mathrm{H}\left(p_{H}\right)+\mathrm{X},(\text { other processes })\right\} \rightarrow\left\{H_{\text {final }}\right\}+X
$$

The hadronic cross-section is given by the factorization theorem as,

$$
\sigma_{\left\{H_{\text {final }}\right\}+X}^{\text {full }}=\sum_{i, j \in \text { partons }} \int d x_{1} d x_{2} f_{i}\left(x_{1}, \mu_{f}\right) f_{j}\left(x_{2}, \mu_{f}\right) \hat{\sigma}_{i j \rightarrow\left\{H_{\text {final }}\right\}+X}^{\text {full }}\left(\hat{s}, \mu_{f}\right)
$$

where

$$
\hat{s}=x_{1} x_{2} s, \quad s \equiv\left(P_{1}+P_{2}\right)^{2} .
$$

The indices $i, j$ run over the flavours of initial state partons. The functions $f_{i}\left(x, \mu_{f}\right)$ are parton distribution functions in the $\overline{\mathrm{MS}}$-factorization scheme and $\mu_{f}$ is the factorization scale.

Singling out typically dominant contributions from resonant diagrams as $p_{H}^{2} \rightarrow m_{H}^{2}$, we cast the partonic cross-section in the form

$$
\hat{\sigma}_{i j \rightarrow\left\{H_{\text {final }}\right\}+X}^{\text {full }}=\hat{\sigma}_{i j \rightarrow\left\{H_{\text {final }}\right\}+X}+\hat{\sigma}_{i j \rightarrow\left\{H_{\text {final }}\right\}+X}^{\text {signal-bkg }}+\hat{\sigma}_{i j \rightarrow\left\{H_{\text {final }}\right\}+X}^{\text {bkg }} .
$$

The first term on the right hand side corresponds to the square of the resonant Feynmandiagrams, the second term corresponds to the interference of resonant and non-resonant diagrams, and the last term is the square of diagrams without a resonant Higgs propagator. We shall refer to the first term as the "signal cross-section". It can be written as:

$$
\hat{\sigma}_{i j \rightarrow\left\{H_{\text {final }}\right\}+X}\left(\hat{s}, \mu_{f}\right)=\int_{Q_{a}^{2}}^{Q_{b}^{2}} d Q^{2} \frac{Q \Gamma_{H}(Q)}{\pi} \frac{\hat{\sigma}_{i j \rightarrow H}\left(\hat{s}, Q^{2}, \mu_{f}\right) \mathrm{Br}_{H \rightarrow\left\{H_{\text {final }}\right\}}(Q)}{\left(Q^{2}-m_{H}^{2}\right)^{2}} .
$$


$Q_{a}, Q_{b}$ define the experimentally accessible range for the invariant mass of the particle system originating from the decay of the intermediate Higgs boson. $m_{H}$ is identified with the Higgs-boson mass. Away from the resonance region, $Q^{2} \sim m_{H}^{2}$ the above equation is adequate for the signal cross-section. iHixs is dedicated to the evaluation of the "signal cross-section". A full description of the cross-section for a Higgs final state can be obtained by adding to the "signal cross-section" the remaining two contributions of eq. (4.4): a theoretical or experimental estimate of the background cross-section and a theoretical estimate of the signal-background interference.

In the resonant limit, $Q^{2} \rightarrow m_{H}^{2}$, the signal cross-section is dominant and it becomes infinite at any fixed order in perturbation theory, for $Q^{2}=m_{H}^{2}$ exactly. A resummation of resonant contributions at all orders is necessary in order to render the propagator finite in this limit. We remark that a resummation of partial perturbative corrections from all perturbative orders into the propagator of an unstable particle is a delicate theoretical issue $[59,60]$. Historically, it has been treated with various prescriptions in the literature with varied success (see, for example, references in [61]). To a first approximation, the signal cross-section becomes:

$$
\hat{\sigma}_{i j \rightarrow\left\{H_{\text {final }}\right\}+X}\left(\hat{s}, \mu_{f}\right)=\int_{Q_{a}^{2}}^{Q_{b}^{2}} d Q^{2} \frac{Q \Gamma_{H}(Q)}{\pi} \frac{\hat{\sigma}_{i j \rightarrow H}\left(\hat{s}, Q^{2}, \mu_{f}\right) \operatorname{Br}_{H \rightarrow\left\{H_{\text {final }}\right\}}(Q)}{\left(Q^{2}-m_{H}^{2}\right)^{2}+m_{H}^{2} \Gamma_{H}^{2}\left(m_{H}\right)} .
$$

$\Gamma_{H}(Q)$ is the decay-width of a Higgs-boson at rest with mass $Q$. In the zero Higgs boson width limit it reduces to the product of the partonic production cross-section $\hat{\sigma}_{i j \rightarrow H}$ for an on-shell Higgs boson times the branching ratio for its decay $\operatorname{Br}_{H \rightarrow\left\{H_{\text {final }}\right\}}$. Performing the transformation

$$
\frac{Q^{2}}{m_{H}^{2}}=1+\delta \tan (\pi y), \quad \delta \equiv \frac{\Gamma_{H}\left(m_{H}\right)}{m_{H}}
$$

we obtain an equivalent integral with a better numerical convergence,

$$
\hat{\sigma}_{i j \rightarrow\left\{H_{\text {final }}\right\}+X}\left(\hat{s}, \mu_{f}\right)=\int_{y_{a}}^{y_{b}} d y \frac{Q \Gamma_{H}(Q)}{m_{H} \Gamma\left(m_{H}\right)} \hat{\sigma}_{i j \rightarrow H}\left(\hat{s}, Q^{2}, \mu_{f}\right) \operatorname{Br}_{H \rightarrow\left\{H_{\text {final }}\right.}(Q) .
$$

The integration boundaries $y_{a, b}$ are computed from eq. (4.7).

A light Higgs boson, as predicted in the Standard Model, has a rather small $\delta$ and it is often sufficient to take the $\delta=0$ limit of the zero width approximation (ZWA). Existing experimental studies at hadron colliders [2-4] have always reported limits on the Higgs boson cross-section comparing with expectations in this approximation. However, recent years have witnessed the alarming trend of using this approximation in situations where it may be severely insufficient. ${ }^{5}$ We therefore find it useful to dedicate a part of our numerical studies to Higgs bosons with a non-negligible width.

The width grows for heavier Higgs boson masses due to decays into electroweak gauge bosons. In extensions of the Standard Model, this feature may be more pronounced as new decay modes may be available. For such situations, the zero width approximation is poor. An integration over the Breit-Wigner distribution of eq. (4.8) is a more accurate estimate

\footnotetext{
${ }^{5}$ For example, recent limits on the cross-section for a heavy Higgs boson [3].
} 
of the signal cross-section. We also note that eq. (4.8) convolutes with the branching ratio. For Higgs masses close to thresholds branching ratios are steeply changing; a naive estimate of the signal cross-section in the zero width approximation could also be unsafe.

iHixs allows the possibility for the calculation of the Higgs signal cross-section taking into account finite Higgs width effects by performing the integral of eq. (4.8). Notice that the resummed expression of eqs. (4.6), (4.8) is in good agreement with eq. (4.5) away from the resonant region only when the partial width, $\Gamma_{H}(Q) \operatorname{Br}_{H \rightarrow\left\{H_{\text {final }}\right\}}(Q)$, is computed at a variable Higgs-boson virtuality $Q$. Higgs decay rates are rather sensitive to the Higgs boson virtuality due to the many decay mechanisms which become available at diverse mass values. In order to use iHixs with an arbitrary BSM model, the user needs to provide a data file with the width and branching ratios of the Higgs boson as a function of the virtuality of the Higgs boson.

For the Standard Model, or models with similar enough width and branching ratios, we have generated a grid of values using the program HDECAY of ref. [26]. ${ }^{6}$ We note that for large Higgs boson widths the description of the Higgs line-shape may require further improvements, both in the resonance region where a more sophisticated resummation framework could be employed [59] and, especially, for virtualities far from the Higgs boson mass where a dedicated estimation of the signal-background interference cross-section is needed. We believe that iHixs can provide a flexible enough platform for such modifications if need arises (e.g. with experimental evidence of a Higgs boson and the associated heavy particles which render the theory consistent with electroweak precision tests and unitarity bounds).

In the Standard-Model, it has been observed that significant cancelations due to interference of resonant and non-resonant diagrams take place at high invariant masses (refs. [62-65]). The magnitude of the "signal-background" cross-section is very important and cannot be neglected. iHixs takes into account only diagrams with an s-channel Higgs boson propagator. The line-shape away from the resonance is therefore poorly described. To improve upon this, we have implemented a prescription based on the resummation of $V V \rightarrow V V$ scattering amplitudes with the dominant contributions from both resonant and non-resonant Feynman diagrams [66] at the high energy regime. The contributions which unitarize the scattering amplitude for vector-boson scattering can be approximated with the amplitude for Goldstone boson scattering at high energies. Conveniently, the amplitude in this regime can be described in terms of an effective Higgs propagator. Ref. [66] performs a Dyson re-summation of the tree-level Goldstone boson scattering amplitude leading to an "improved s-channel approximation". In this framework, the Higgs propagator is modified according to the prescription:

$$
\frac{i}{\hat{s}-m_{H}^{2}} \rightarrow \frac{i \frac{m_{H}^{2}}{\hat{s}}}{\hat{s}-m_{H}^{2}+i \Gamma_{H}\left(m_{H}^{2}\right) \frac{\hat{s}}{m_{H}}} .
$$

This prescription interpolates smoothly between two limits which are well described either by resummation or by fixed-order perturbation theory: the resonant region $Q \sim m_{H}$

\footnotetext{
${ }^{6}$ For studies with iHixs where the HDECAY tabulated Higgs boson width and branching ratios are used we request that ref. [26] is also cited.
} 
and the high energy limit $Q \gg m_{H}$. We do not envisage eq. (4.9) as the final step towards a precise description of the line-shape for the Higgs boson. However, it is a very useful diagnostic tool in order to assess how important the signal-background interference could be for a heavy Higgs boson. According to this prescription, the hadronic cross-section is computed as,

$$
\begin{aligned}
\hat{\sigma}_{i j \rightarrow\left\{H_{\text {final }}\right\}+X}\left(\hat{s}, \mu_{f}\right)= & \int_{y_{a}}^{y_{b}} d y \frac{Q \Gamma_{H}(Q)}{m_{H} \Gamma\left(m_{H}\right)} \hat{\sigma}_{i j \rightarrow H}\left(\hat{s}, Q^{2}, \mu_{f}\right) \operatorname{Br}_{H \rightarrow\left\{H_{\text {final }}\right\}}(Q) \\
& \times f_{\text {seym }}\left(Q, m_{H}\right),
\end{aligned}
$$

with

$$
f_{\text {seym }}\left(Q^{2}, m_{H}^{2}\right) \equiv \frac{m_{H}^{4}}{Q^{4}} \frac{\left(1-\frac{Q^{2}}{m_{H}^{2}}\right)^{2}+\delta^{2}}{\left(1-\frac{Q^{2}}{m_{H}^{2}}\right)^{2}+\delta^{2} \frac{Q^{4}}{m_{H}^{4}}}
$$

We emphasize once again that when eq. (4.10) is used (Seymour option in iHixs) some signal-background interference effects which are dominant at very high invariant masses are taken into account. In contrast, eq. (4.8) (default option in iHixs) computes purely the signal cross-section (only the square of resonant Feynman diagrams).

We believe that the Higgs boson line-shape will enjoy many future theoretical studies with improved resummation methods for resonant diagrams and matching to fixed-order perturbation theory away from the resonance region. In the course of these developments, new prescriptions which are not yet implemented in iHixs shall emerge. We have made efforts to be able to include such improvements readily in iHixs. For example, a method introduced recently in ref. [67] requires that all virtual amplitudes must be computed with a complex Higgs virtuality $Q^{2}$. This requirement is effortless to achieve in iHixs where we evaluate all QCD one and two-loop amplitudes using CHAPLIN [18] for harmonic polylogarithms with complex arguments. We defer to the future a conceptual appraisal and a numerical comparison of theoretically appealing approaches beyond the prescriptions of eq. (4.8) and eq. (4.10).

We now define the dimensionless ratios

$$
\tau \equiv \frac{m_{H}^{2}}{s} \quad \hat{\tau} \equiv \frac{Q^{2}}{s} \quad z \equiv \frac{\hat{\tau}}{x_{1} x_{2}}
$$

The hadronic Higgs signal cross-section can be cast in the form

$$
\begin{aligned}
\sigma_{\left\{H_{\text {final }}\right\}+X}= & \sum_{i, j \in \text { partons }} \int_{y_{a}}^{y_{b}} d y \frac{Q \Gamma_{H}(Q)}{m_{H} \Gamma\left(m_{H}\right)} \operatorname{Br}_{H \rightarrow\left\{H_{\text {final }}\right\}}(Q) f_{\text {seym }}\left(Q, m_{H}\right) \times \\
& \times \sum_{i j} \int \frac{d x}{x} d z \mathcal{L}_{i j}\left(x_{1}, x_{2}, \mu_{f}\right)\left[\frac{\hat{\sigma}_{i j \rightarrow H}\left(Q^{2}, z, \mu_{f}\right)}{z}\right]
\end{aligned}
$$

where

$$
x_{1} \equiv x, \quad x_{2} \equiv \frac{\hat{\tau}}{x z}, \quad \mathcal{L}_{i j} \equiv\left[x_{1} f_{i}\left(x_{1}\right)\right]\left[x_{2} f_{j}\left(x_{2}\right)\right] .
$$

In iHixs we have implemented the partonic cross-sections for two Higgs boson production channels: 
(i) bottom-quark fusion as in ref. [57]

(ii) gluon fusion.

Amplitudes for the two processes do not interfere and the two cross-sections can be computed independently.

We have faithfully adopted the definitions and analytic expressions for the partonic quark-fusion cross-sections of ref. [57], where they have been computed through NNLO in the strong coupling expansion. We shall therefore not discuss this process any further, except for the presentation of numerical results with iHixs.

The perturbative evaluation of the gluon fusion cross-section has been the topic of numerous publications. Nevertheless, for the sake of clarity, we find it important to elaborate on our implementation of perturbative corrections for this process.

\section{The gluon fusion process through NLO QCD}

We cast the partonic cross-sections in the form

$$
\frac{\hat{\sigma}_{i j \rightarrow H}\left(Q^{2}, z, \mu_{f}\right)}{z}=\frac{G_{f} \pi}{288 \sqrt{2}} \sum_{p=0}^{\infty}\left(\frac{\alpha_{s}\left(\mu_{r}\right)}{\pi}\right)^{2+p} n_{i j}^{(p)}\left(Q^{2}, z, \mu_{f}, \mu_{r}\right),
$$

where $\mu_{r}$ is the renormalization scale for the strong coupling.

The partonic cross sections $z n_{i j}^{(p)}$ at NLO and higher orders consist of virtual and real emission parts that are separately infrared divergent. We expose the infrared singularities of the real radiation matrix-elements by the method of plus-distribution subtractions. We then add the virtual part, the collinear counter term which has been generated from parton distribution factorization in the $\overline{\mathrm{MS}}$-factorization scheme and the renormalization counter terms in the $\overline{\mathrm{MS}}$-renormalization scheme. The resulting cross-section is finite and can be written as the sum of three distinct terms: a term proportional to $\delta(1-z)$ that corresponds to all contributions from leading order kinematics (virtual part plus $\delta$-proportional terms from the integrated soft-collinear pieces), a 'regular' term that corresponds to higher order kinematics, and plus-distribution pieces, proportional to $\left[\frac{f(z)}{1-z}\right]_{+}$for various $f(z)$.

We therefore write

$$
n_{i j}^{(p)}\left(Q^{2}, z, \mu_{f}, \mu_{r}\right)=\sum_{k \in\{\delta,+, R\}} n_{i j ; k}^{(p)}\left(Q^{2}, z, \mu_{f}, \mu_{r}\right)
$$

$5.1 \quad$ LO: $g g \rightarrow h$

At leading order only the gluon gluon subprocess contributes. One obtains

$$
n_{g g ; \delta}^{(0)}=\left|\sum_{q} Y_{q} \tau_{q} \frac{3}{2} A\left(\tau_{q}\right)\right|
$$

with

$$
\tau_{q} \equiv \frac{4 m_{q}\left(m_{q}-i \Gamma_{q}\right)}{Q^{2}}
$$


and $m_{q}$ being the mass of the heavy quark in the gluon fusion loop. The quantity $\tau_{q} \frac{3}{2} A\left(\tau_{q}\right)$ has the simple limits

$$
\lim _{\tau_{q} \rightarrow \infty} \tau_{q} \frac{3}{2} A\left(\tau_{q}\right)=1 \quad \lim _{\tau_{q} \rightarrow 0} \tau_{q} \frac{3}{2} A\left(\tau_{q}\right)=0
$$

The full analytic expression is given by,

$$
A\left(\tau_{q}\right)=1-\frac{1}{2} \frac{\left(1+x_{q}\right)^{2}}{\left(1-x_{q}\right)^{2}} H\left(0,0 ; x_{q}\right)
$$

with

$$
x_{q}=\frac{-\tau_{q}}{\left(\sqrt{1-\tau_{q}}+1\right)^{2}}
$$

Note that the sum runs over all quarks in the model, and $Y_{q}$ is as defined in the Feynman rules of section 3 .

\section{$5.2 \quad$ NLO: $g g \rightarrow h+g$}

The $\delta$-part of the NLO correction to the gluon gluon subprocess can be written as

$$
n_{g g ; \delta}^{(1)}=|B|^{2}\left[2 \beta_{0} \log \left(\frac{\mu_{R}^{2}}{\mu_{F}^{2}}\right)+\pi^{2}\right]+\Re\left[B \sum_{q} V_{q}\left(\tau_{q}\right)^{*}\right]
$$

where

$$
B \equiv \sum_{q} Y_{q} \tau_{q} \frac{3}{2} A\left(\tau_{q}\right)
$$

is the LO coefficient, and

$$
V_{q}\left(\tau_{q}\right)=Y_{q} \frac{3}{8} M_{\text {fin }}^{(1)}\left(\tau_{q}\right)=Y_{q} \frac{\mathcal{G}_{i}^{2 l}}{-2 / 3}
$$

where $M_{\text {fin }}^{(1)}\left(\tau_{q}\right)$ can be found in eq. (7.4) of ref. [32] and $\mathcal{G}_{i}^{2 l}$ can be found in eq. (26)-(30) of ref. [33]. The plus distribution part is

$$
n_{g g ;+}^{(1)}=|B|^{2}\left[-6 \log \left(\frac{\mu_{F}^{2}}{Q^{2}}\right)\left[\frac{1}{1-z}\right]_{+}+12\left[\frac{\log (1-z)}{1-z}\right]_{+}\right]
$$

Finally, the regular part of the NLO gluon gluon correction is

$$
\begin{aligned}
n_{g g ; R}^{(1)}= & \frac{3}{z(1-z) \lambda(1-\lambda)}\left\{\frac{1}{2} z^{4} \sum_{j=1}^{4}\left|\sum_{q} A_{g g g H}^{j q}\left(\tau_{q}\right)\right|^{2}-\left(1-z+z^{2}\right)^{2}|B|^{2}\right\} \\
& +|B|^{2}\left\{6\left[p_{g g}(z) \log \left(\frac{(1-z)^{2}}{z}\right)-\log \left(\frac{z}{1-z}\right)\right]-6 \log \left(\frac{\mu_{F}^{2}}{Q^{2}}\right) p_{g g}(z)\right\}
\end{aligned}
$$

with $p_{g g}(z)$ the gluon splitting kernel

$$
p_{g g}(z)=\frac{1}{z}+z(1-z)-2
$$

The form factors $A_{g g g H}^{j q}\left(\tau_{q}\right)$ can be found in the appendix B.2. 


\subsection{NLO: $q \bar{q} \rightarrow h+X$}

The $q \bar{q}$ initial state starts contributing to the total cross section at order $a_{s}^{3}$ in QCD. There are also non-negligible mixed QCD-electroweak corrections, at order $a_{s}^{2} a_{\text {ewk }}$ which we shall discuss later. The pure QCD corrections lead to the following coefficients:

$$
\begin{aligned}
n_{q \bar{q} ; \delta}^{(1)} & =n_{q \bar{q} ;+}^{(1)}=0 \\
n_{q \bar{q} ; R}^{(1)} & =\frac{32}{27} \frac{(1-z)^{3}}{z}\left|\sum_{q} Y_{q} \tau_{q} A_{q \bar{q} g H}(z)\right|^{2}
\end{aligned}
$$

with $A_{q \bar{q} g H}(z)$ given in eq. (B.5).

\subsection{NLO: $q g \rightarrow h+X$}

The gluon-quark initial state also contributes at order $a_{s}^{3}$, and it receives similar mixed QCD-electroweak corrections of order $a_{s}^{2} a_{\text {ewk }}$. The pure QCD coefficient is:

$$
n_{q g ; \delta}^{(1)}=n_{q g ;+}^{(1)}=0,
$$

and

$$
\begin{aligned}
n_{q g ; R}^{(1)}= & \left\{|B|^{2}\left[\frac{C_{F}}{2} z-p_{g q}(z) \log \left(\frac{z}{(1-z)^{2}}\right)-p_{g q}(z) \log \left(\frac{\mu_{F}^{2}}{Q^{2}}\right)\right]\right. \\
& \left.+\int_{0}^{1} d \lambda \frac{1}{(1-\lambda)_{+}}\left[\left|\sum_{q} Y_{q} \tau_{q} A_{q \bar{q} g H}\left(y_{\lambda}\right)\right|^{2} \frac{1+(1-z)^{2} \lambda}{z}\right]\right\}
\end{aligned}
$$

with

$$
\begin{aligned}
y_{\lambda} & =\frac{-z}{(1-z)(1-\lambda)} \\
p_{g q}(z) & =\frac{C_{F}}{2} \frac{1+(1-z)^{2}}{z}
\end{aligned}
$$

and $A_{q \bar{q} g H}(z)$ given in eq. (B.5).

\subsection{NLO: mixed QCD-EW corrections to $q \bar{q} \rightarrow H+g$ and $q g \rightarrow h+g$}

The mixed QCD-electroweak contributions ${ }^{7}$ have the following structure:

$$
\begin{aligned}
n_{q \bar{q} ; R}^{(0) \text { ewk }}= & \lambda_{\text {ewk }} \frac{8}{3} \frac{1-z}{z} \sum_{q} \sum_{X \in\left\{W_{i}, Z_{i}, H\right\}} \Re\left\{\tau_{q} A_{q q g H}(z) c_{X, q} .\right. \\
& {\left.\left[F_{1, X, q}^{*}\left(s_{13}, s_{23}, s_{12}\right) \frac{s_{13}^{2}}{s_{12}}+F_{2, X, q}^{*}\left(s_{13}, s_{23}, s_{12}\right) \frac{s_{23}^{2}}{s_{12}}\right]\right\} }
\end{aligned}
$$

where $A_{q \bar{q} g H}(z)$ given in eq. (B.5). The sum over $X$ runs over all $W$ and $Z$-like bosons in the model, as well as over the Higgs boson, while the sum over $q$ runs over all heavy quarks, $b, t, \ldots$, as before. The coupling $c_{X, q}$ contains Kronecker delta symbols which select specific initial state quarks, depending on $X$, as explained below.

\footnotetext{
${ }^{7}$ Note that these corrections are of order $a_{s}^{2}$, so we denote them by $n_{q \bar{q}}^{(0) \text { ewk }}$.
} 
- $Z$ loops: for the initial state quarks $q \in\{u, d, c, s, b\}$ we have the following couplings

$$
\begin{array}{rlrl}
\lambda_{Z} & =2 & \\
c_{Z, q} & =\left(\delta_{q u}+\delta_{q c}\right)\left(v_{Z, u}^{2}+a_{Z_{u}}^{2}\right)+\left(\delta_{q d}+\delta_{q s}+\delta_{q b}\right)\left(v_{Z, d}^{2}+a_{Z_{d}}^{2}\right) \\
v_{Z, u} & =\frac{g_{w}}{\cos \theta_{w}}\left(\frac{1}{2}-\frac{4}{3} \sin ^{2} \theta_{w}\right), & a_{Z, u}=\frac{g_{w}}{2 \cos \theta_{w}} \\
v_{Z, d}=\frac{g_{w}}{\cos \theta_{w}}\left(-\frac{1}{2}+\frac{2}{3} \sin ^{2} \theta_{w}\right), & a_{Z, d}=\frac{g_{w}}{2 \cos \theta_{w}}
\end{array}
$$

The form factor is identical for all initial state quarks and yields

$$
\begin{aligned}
& \mathcal{F}_{1}^{Z_{q}}=-m_{z}^{2} A_{\text {ewk }}\left(s_{31}, s_{23}, s_{12}, Q, m_{z}\right) \\
& \mathcal{F}_{2}^{Z_{q}}=-m_{z}^{2} A_{\text {ewk }}\left(s_{23}, s_{31}, s_{12}, Q, m_{z}\right)
\end{aligned}
$$

with $A_{\text {ewk }}\left(s, t, u, m_{h}, m_{z}\right)$ given in the appendix in eq. (B.7).

- W loops: the couplings are given by

$$
\begin{aligned}
& \lambda_{W}=2, \quad c_{W, q}=\left(v_{W, q}^{2}+a_{W, q}^{2}\right), \quad v_{W, q}=\frac{g_{w}}{\sqrt{2}}, \quad a_{W, q}=\frac{g_{w}}{\sqrt{2}}, \\
& \mathcal{F}_{1}^{W}=-m_{w}^{2} A_{\text {ewk }}\left(s_{31}, s_{23}, s_{12}, Q^{2}, m_{w}\right) \sum_{L} \delta_{q L}-m_{w}^{2} A_{\mathrm{ewk}}^{m_{t}}\left(s_{31}, s_{23}, s_{12}\right) \delta_{q b} \\
& \mathcal{F}_{2}^{W}=-m_{w}^{2} A_{\mathrm{ewk}}\left(s_{23}, s_{31}, s_{12}, Q^{2}, m_{w}\right) \sum_{L} \delta_{q L}-m_{w}^{2} A_{\mathrm{ewk}}^{m_{t}}\left(s_{23}, s_{31}, s_{12}\right) \delta_{q b}
\end{aligned}
$$

where $L$ sums over all light quark states $u, d, c, s$. Note that here we have summed over the internal light quark flavors. This yields a CKM coefficient of

$$
\sum_{j=1,2}\left|V_{i j}\right|^{2} \approx 1 \quad \text { for } i=1,2
$$

Measurements show this to be true to about 1 in 10000 , so it is a good enough approximation to make.

For $q \in\{b\}$, we have to take the internal quark to be a top. The couplings are unchanged if we use that $\left|V_{33}\right|^{2} \approx 1$, which is also a good approximation to make. The form factor $A_{\mathrm{ewk}}^{m_{t}}(s, t, u)$ is given in the appendix in eq. (B.9).

- Higgs in the loop: in the standard model this gives a non negligible contribution for $q=b$, other quarks may be considered as well if their Yukawas are enhanced. The couplings are given by

$$
\lambda_{H}=3, \quad c_{H, q}=\delta_{q b}\left(v_{H, q}^{2}+a_{H, q}^{2}\right), \quad v_{H, q}=\frac{m_{q} Y_{q}}{v}, \quad a_{H, q}=0,
$$

and the form factors are

$$
\begin{aligned}
& \mathcal{F}_{1}^{H}=-m_{h}^{2} A_{H}\left(s_{31}, s_{23}, s_{12}\right) \\
& \mathcal{F}_{2}^{H}=-m_{h}^{2} A_{H}\left(s_{23}, s_{31}, s_{12}\right) .
\end{aligned}
$$




\section{Beyond the NLO QCD}

The leading order and next to leading oder cross-section are known exactly. Beyond that we can only take the limit of heavy electroweak gauge bosons and top-quarks. Bottom quark contributions are also unknown beyond NLO.

In the effective theory approximation, the Higgs gluon interaction is described by an operator of the form

$$
\mathcal{L}_{\text {eff }}=-\frac{1}{3 \pi} C_{w} \cdot H G_{\mu \nu} G^{\mu \nu},
$$

where the Wilson coefficient has a perturbative expansion

$$
C_{w}=C_{0}+C_{1} \frac{\alpha_{s}(\mu)}{\pi}+C_{2}\left(\frac{\alpha_{s}(\mu)}{\pi}\right)^{2}+\ldots
$$

The cross-section calculated in the effective theory is,

$$
\sigma_{\mathrm{eff}}=\left|C_{0}+C_{1} \frac{\alpha_{s}(\mu)}{\pi}+C_{2}\left(\frac{\alpha_{s}(\mu)}{\pi}\right)^{2}+\ldots\right|^{2}\left[\eta_{0}+\frac{\alpha_{s}}{\pi} \eta_{1}+\left(\frac{\alpha_{s}}{\pi}\right)^{2} \eta_{2}+\ldots\right]
$$

Expanding in $\alpha_{S}$ we obtain

$$
\sigma_{\mathrm{eff}}=\sigma_{\mathrm{eff}}^{(0)}+\left(\frac{\alpha_{s}}{\pi}\right) \sigma_{\mathrm{eff}}^{(1)}+\left(\frac{\alpha_{s}}{\pi}\right)^{2} \sigma_{\mathrm{eff}}^{(2)}+\ldots
$$

with

$$
\begin{aligned}
\sigma_{\text {eff }}^{(0)} & =\left|C_{0}\right|^{2} \eta_{0}, \\
\sigma_{\text {eff }}^{(1)} & =\left|C_{0}\right|^{2} \eta_{1}+2 \operatorname{Re}\left(C_{0} C_{1}\right) \eta_{0}, \\
\sigma_{\text {eff }}^{(2)} & =\left|C_{0}\right|^{2} \eta_{2}+2 \operatorname{Re}\left(C_{0} C_{1}\right) \eta_{1}+\left(\left|C_{1}\right|^{2}+2 \operatorname{Re}\left(C_{0} C_{2}\right)\right) \eta_{0} .
\end{aligned}
$$

The integrated cross-sections $\eta_{i}$ have been computed through NNLO in refs. [43-45].

Resorting to an effective theory calculation is necessary only for important QCD and electroweak corrections which cannot be evaluated in the full theory. In the previous sections, we have listed results in the exact theory for the LO and NLO QCD perturbative expansion as well as one-loop electroweak corrections. In addition, two-loop electroweak corrections are also known exactly [47]. We will keep these corrections with their full mass dependence as in the exact theory calculations and use the effective theory approach for contributions at higher orders in the strong and electroweak couplings, namely for the NNLO correction in QCD and mixed QCD and electroweak corrections.

We match the effective theory and full theory perturbative expansions as follows. Let us assume that we can compute the contributions to the cross-section exactly through some perturbative order for only some of the heavy particles which contribute to Higgs production amplitudes:

$$
\sigma_{\text {partial }}=\sigma_{\text {partial }}^{(0)}+\left(\frac{\alpha_{s}}{\pi}\right) \sigma_{\text {partial }}^{(1)}+\left(\frac{\alpha_{s}}{\pi}\right)^{2} \sigma_{\text {partial }}^{(2)}+\ldots
$$


In an effective theory approach, these contributions would factorize as in:

$\sigma_{\text {partial }, \text { eff }}=\left|C_{0}^{\text {partial }}+C_{1}^{\text {partial }} \frac{\alpha_{s}(\mu)}{\pi}+C_{2}^{\text {partial }}\left(\frac{\alpha_{s}(\mu)}{\pi}\right)^{2}+\ldots\right|^{2}\left[\eta_{0}+\frac{\alpha_{s}}{\pi} \eta_{1}+\left(\frac{\alpha_{s}}{\pi}\right)^{2} \eta_{2}+\ldots\right]$

We then write the cross-section as

$$
\sigma=\sum_{n=0}^{\infty}\left(\frac{\alpha_{s}}{\pi}\right)^{n}\left[\sigma_{\text {partial }}^{(n)} \Theta(n \leq \text { Norder })+\delta \sigma_{\text {eff }}\right]
$$

where Norder is the last perturbative order that the partial contributions are known in the full theory and

$$
\begin{aligned}
\delta \sigma_{\text {eff }}^{(0)}= & \left\{\left|C_{0}\right|^{2}-\Theta(0 \leq \text { Norder })\left|C_{0}^{\text {partial }}\right|^{2}\right\} \eta_{0}, \\
\delta \sigma_{\text {eff }}^{(1)}= & \left\{\left|C_{0}\right|^{2}-\Theta(1 \leq \text { Norder })\left|C_{0}^{\text {partial }}\right|^{2}\right\} \eta_{1} \\
& +\left\{2 \operatorname{Re}\left(C_{0} C_{1}\right)-\Theta(1 \leq \text { Norder }) 2 \operatorname{Re}\left(C_{0}^{\text {partial }} C_{1}^{\text {partial }}\right)\right\} \eta_{0}, \\
\delta \sigma_{\text {eff }}^{(2)}= & \left\{\left|C_{0}\right|^{2}-\Theta(2 \leq \text { Norder })\left|C_{0}^{\text {partial }}\right|^{2}\right\} \eta_{2} \\
& +\left\{2 \operatorname{Re}\left(C_{0} C_{1}\right)-\Theta(2 \leq \text { Norder }) 2 \operatorname{Re}\left(C_{0}^{\text {partial }} C_{1}^{\text {partial }}\right)\right\} \eta_{1} \\
& +\left(\left\{\left|C_{1}\right|^{2}-\Theta(2 \leq \text { Norder })\left|C_{1}^{\text {partial }}\right|^{2}\right\}\right. \\
& \left.+\left\{2 \operatorname{Re}\left(C_{0} C_{2}\right)-\Theta(2 \leq \text { Norder }) 2 \operatorname{Re}\left(C_{0}^{\text {partial }} C_{2}^{\text {partial }}\right)\right\}\right) \eta_{0} .
\end{aligned}
$$

\subsection{The Standard Model Wilson coefficient with anomalous Yukawa and elec- troweak couplings}

In our theory, we can integrate out the top-quark, the new heavy quarks, and the electroweak gauge bosons $\mathrm{W}$ and $\mathrm{Z}$. This yields a Wilson coefficient which is

$$
\begin{aligned}
& C_{0}=\lambda_{\mathrm{QCD}} \cdot 1+\lambda_{\mathrm{EWK}} \cdot 1 \\
& C_{1}=\lambda_{\mathrm{QCD}} \cdot \frac{11}{4}+\lambda_{\mathrm{EWK}} \cdot \frac{7}{6} \\
& C_{2}=\lambda_{\mathrm{QCD}} \cdot C_{2 q}+\lambda_{\mathrm{EWK}} \cdot C_{2 w} .
\end{aligned}
$$

The factor for the electroweak component is given by

$$
\lambda_{\mathrm{EWK}}=\lambda_{\mathrm{ewk}} \frac{3 \alpha}{16 \pi s_{w}^{2}}\left\{4+\frac{2}{c_{w}^{2}}\left[\frac{5}{4}-\frac{7}{3} s_{w}^{2}+\frac{22}{9} s_{w}^{4}\right]\right\}
$$

with $s_{w}, c_{w}$ the sine and the cosine of the Weinberg mixing angle. The factor for the QCD component is given by the sum of the anomalous Yukawa coupling re-scaling factors for all heavy quarks

$$
\lambda_{\mathrm{QCD}}=\sum_{q \in \text { heavy }} Y_{q} .
$$

The three-loop QCD Wilson coefficient $C_{2 q}$ has been computed recently in ref. [11] and can be read from eq. (3.35) of the same reference. The four-loop electroweak Wilson coefficient is not yet known.

$$
C_{2 w}=\text { unknown }
$$


One can attempt a rough estimation since it is conceivable that the perturbative series for the QCD component and the electroweak component follow a similar pattern: $\left|C_{2 w}\right| \sim$ $\left|C_{2 q}\right| \sim 10$. In our studies, we vary $C_{2 w} \in[-30,30]$ as an estimate of the higher order mixed QCD and electroweak corrections.

In iHixs we have implemented the exact contributions up to NLO, i.e. Norder $=1$, for the heavy quarks and the electroweak gauge-bosons. These contributions should not be counted twice in the effective theory calculation.

$$
\begin{aligned}
& C_{0}^{\text {partial }}=\lambda_{\mathrm{QCD}} \cdot 1 \\
& C_{1}^{\text {partial }}=\lambda_{\mathrm{QCD}} \cdot \frac{11}{4} \\
& C_{2}^{\text {partial }}=\lambda_{\mathrm{QCD}} \cdot C_{2 q} .
\end{aligned}
$$

\subsection{Improving on the effective theory approximation}

It has been observed that the effective theory works better for the K-factors rather than the absolute cross-section. At next-to-leading order, all real and virtual amplitudes in the soft or collinear limit have the same dependence on the masses of the heavy quarks as at leading order. It appears that the factorization of the cross-section in the infrared limit closely resembles the factorization of the cross-section in the limit of infinitely heavy massive particles. Finite quark-mass effects are important for "hard radiation" terms, but these are expected to have a typical perturbative expansion where an $\alpha_{s}$ suppression occurs from one order to the other. Top-quark mass effects have been studied with explicit calculations with operators of higher dimension in HQET demonstrating the validity of the approach at NNLO [70,71]. We can then improve on the effective theory approximation by making the replacement

$$
\lambda_{\mathrm{QCD}} \rightarrow \sum_{q \in \text { heavy }} Y_{q} \frac{3}{2} \tau_{q} A\left(\tau_{q}\right)
$$

and

$$
\lambda_{\mathrm{EWK}} \rightarrow \lambda_{\mathrm{ewk}} \mathcal{M}_{g g h, \mathrm{EWK}}\left(m_{H}^{2}, M_{W}^{2}, M_{Z}^{2}, \ldots\right)
$$

the two-loop electroweak amplitude for $g g \rightarrow H$, computed fully in [46, 47]. In figure 21 of ref. [46] we find the quantity

$$
\delta_{\mathbf{E W K}} / 100=\left.\frac{\sigma_{\mathrm{EWK}+\mathrm{QCD}}^{\text {born }}}{\sigma_{\mathrm{QCD}}^{\text {born }}}\right|_{\text {top only }}-1=\left|1+\frac{\mathcal{M}_{g g h, \mathrm{EWK}}}{\frac{3}{2} \tau_{q} A\left(\tau_{\mathrm{top}}\right)}\right|^{2}-1 .
$$

We then substitute,

$$
\lambda_{\mathrm{EWK}} \rightarrow \lambda_{\mathrm{ewk}} \frac{3}{2} \tau_{q} A\left(\tau_{\mathrm{top}}\right) \times \lambda_{0},
$$

with

$$
\lambda_{0}=\sqrt{1+\frac{\delta_{\mathrm{EWK}}}{100}}-1 .
$$

The two-loop electroweak corrections were kindly provided to us in a data file, electroweak.h, by the authors of ref. [46]. 


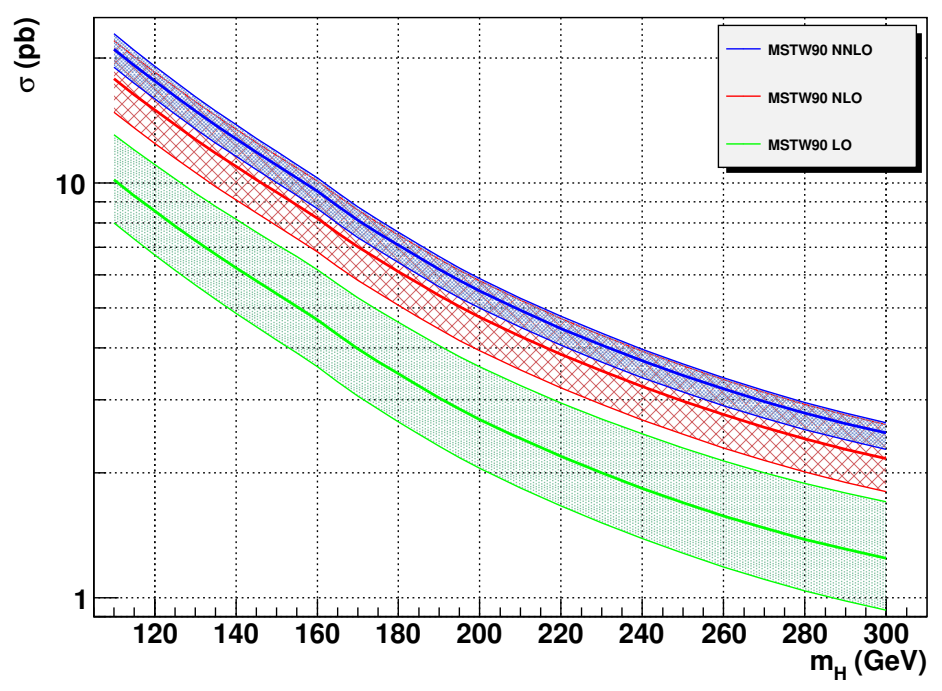

Figure 1. Inclusive Higgs cross section at LO, NLO and NNLO, with scale uncertainty bands, calculated in the range $\mu \in\left[\mu_{0} / 2,2 \mu_{0}\right]$ for MSTW PDFS.

\section{Numerical results in gluon fusion}

In this section, we present numerical results for the Higgs boson cross-section via gluon fusion. We will first make a short discussion of the stability of the perturbative expansion and the scale variation uncertainty. Then we shall compare predictions from all available NNLO sets of parton distribution functions. We will proceed with a study of finite width effects for the heavy quarks in the gluon fusion loops. Finally we shall discuss the finite width effects on the Higgs boson total cross-section. To the best of our knowledge, there have been no published result for NNLO K-factors for the signal cross-section beyond the zero width approximation for the Higgs boson.

\subsection{Perturbative convergence and scale uncertainty}

The gluon fusion cross-section exhibits a rather slow convergence of the perturbative series in the strong coupling constant. iHixs computes the cross-section through NNLO in perturbative QCD. The perturbative behavior of the total higgs cross section with its scale uncertainty, when using MSTW08 parton densities, is shown in figure 1 for LHC at $7 \mathrm{TeV}$ collision energy. One notices that radiative corrections are sizable, where neither the NLO nor the NNLO corrections can be neglected.

A major source of theoretical uncertainty in Higgs production via gluon fusion is due to the choice of the factorization and renormalization scales. We evaluate the scale uncertainty with a central scale of $\mu_{F}=\mu_{R}=\mu_{0}=m_{H} / 2$, and a variation in the range $\mu \in\left[\mu_{0} / 2,2 \mu_{0}\right]$. We note that the NLO scale uncertainty band engulfs the NNLO band in almost the entire mass region depicted. We also notice that the magnitude of the perturbative corrections (K-factor) is larger for lower values of the Higgs boson mass.

Similar behavior is observed when using the ABKM set, but not with the GJR set, figure 2, where the NLO and NNLO overlap is only partial. 

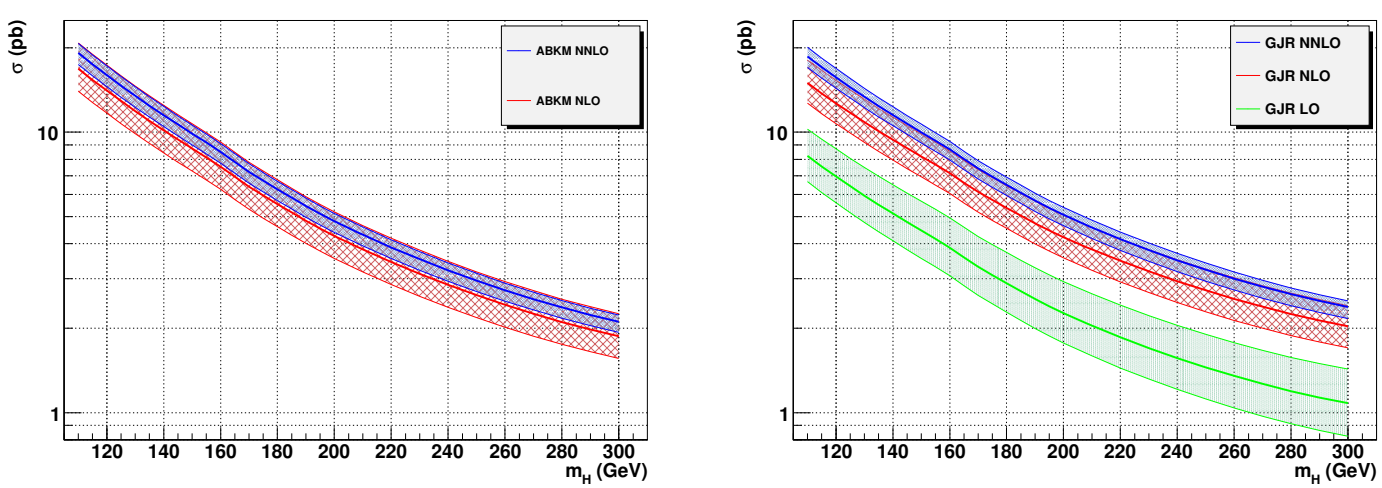

Figure 2. Inclusive Higgs cross section at LO, NLO and NNLO, with scale uncertainty bands, calculated in the range $\mu \in\left[\mu_{0} / 2,2 \mu_{0}\right]$ for ABKM and GJR PDF sets.

\subsection{PDF comparison}

An enormous progress has been made in the last decade towards improving and estimating reliably the precision of parton densities. While uncertainties are generally small, the gluon fusion process requires the less constrained gluon density. The extraction of this quantity is an active field of research. It is very important to compare the effect of various gluon density determinations on the Higgs boson cross-section. We have enabled the possibility for such studies in iHixs.

In tables 5, 6, 7 we present the inclusive cross section for $p p \rightarrow H+X$ for Higgs masses ranging from 110 to $300 \mathrm{GeV}$, using three different PDF sets available at NNLO, namely, MSTW2008 [24], ABKM09 [23] and GJR09 [25]. We have restricted our choice of pdf sets to the ones with NNLO DGLAP evolution, consistently with the iHixs computation of the partonic cross-sections through the same order. We believe that these iHixs results constitute the most precise predictions in fixed order perturbation theory for the Higgs boson cross-section.

A comparison between the three different NNLO PDF sets and the corresponding $\mathrm{PDF}+\alpha_{s}$ uncertainty bands are shown in figure 3. The uncertainties quoted include variations of $\alpha_{s}$ around the preferred value for every set, and are estimated according to the prescriptions of the PDF providers. A combination of cross-section values for a large set of pdf parameterizations is necessary, and these are efficiently computed in iHixs simultaneously.

The reason for the remarkably different predictions between the different PDF sets (that range from $10 \%$ in the low mass region to $30 \%$ in moderately high masses of around $300 \mathrm{GeV}$ ) is hard to trace. The bulk of it may be attributed to differences in the adopted values of the strong coupling constant $a_{s}\left(m_{Z}\right)$. The situation is only partially remedied if one chooses to consider the 90\% CL (as opposed to the one-sigma, 68\% CL) uncertainty bands provided by the MSTW collaboration.

The comparison, in figure 4, shows the ABKM and MSTW uncertainty bands to marginally overlap. We finally note that preliminary results [68] with the updated ABM10 PDF fit, which includes hadron collider data, show that larger values for the total cross section are obtained, in comparison with ABKM09. 

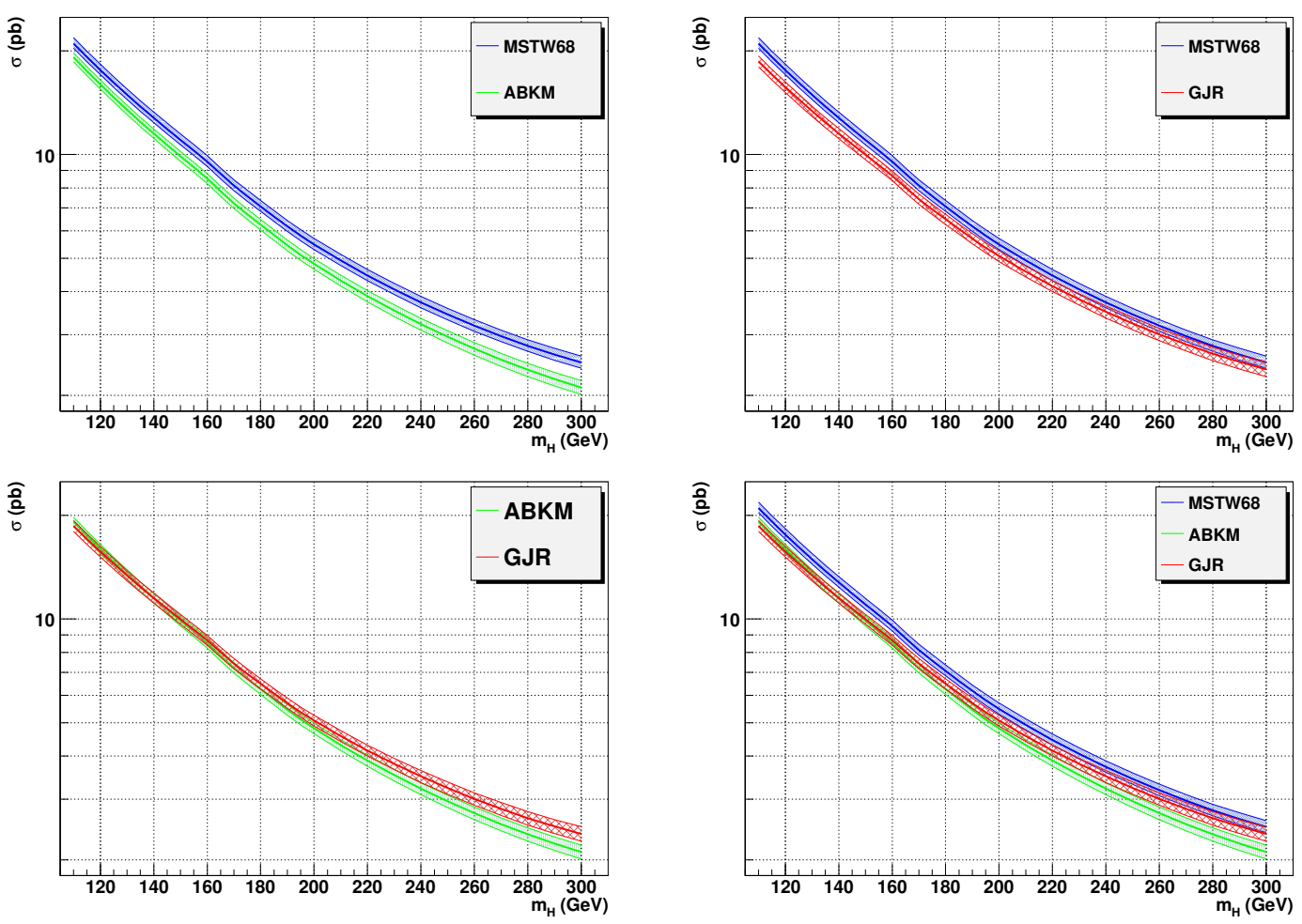

Figure 3. The Higgs production cross section at NNLO with three different PDF sets and their $68 \%$ CL PDF $+a_{s}$ uncertainty bands.

\subsection{Top quark width}

iHixs evaluates two and one-loop amplitudes in all kinematic regions, permiting a definition of mass and kinematic invariants in the full complex plane. This is a particularly useful feature when a resummation of finite width effects in threshold regions is necessary. For example, in a resummation framework using the complex mass scheme [60,67] the masses of heavy quarks need to be evaluated according to the prescription,

$$
m_{q}^{2} \rightarrow m_{q}\left(m_{q}-i \Gamma_{q}\right)
$$

where $\Gamma_{q}$ is the total decay width of the quark and $m_{q}$ its mass.

Using iHixs, we have studied the finite width effects for quarks in fermion loops. We find that the top width is insignificant (at the level of less than one per mille) for a Higgs boson mass below the $t \bar{t}$ threshold. Around and above that threshold, its effect grows to the percent order as shown in figure 5 .

\subsection{Finite Higgs boson width effects}

In section 4 we discussed that there exist various approaches on how to treat the Higgs propagator when departing from the zero width approximation (ZWA). In this section we will present numerical results for the two different prescriptions described in section 4: the default scheme (DEF) of eq. (4.8) and the Seymour scheme (S) of eq. (4.10). 

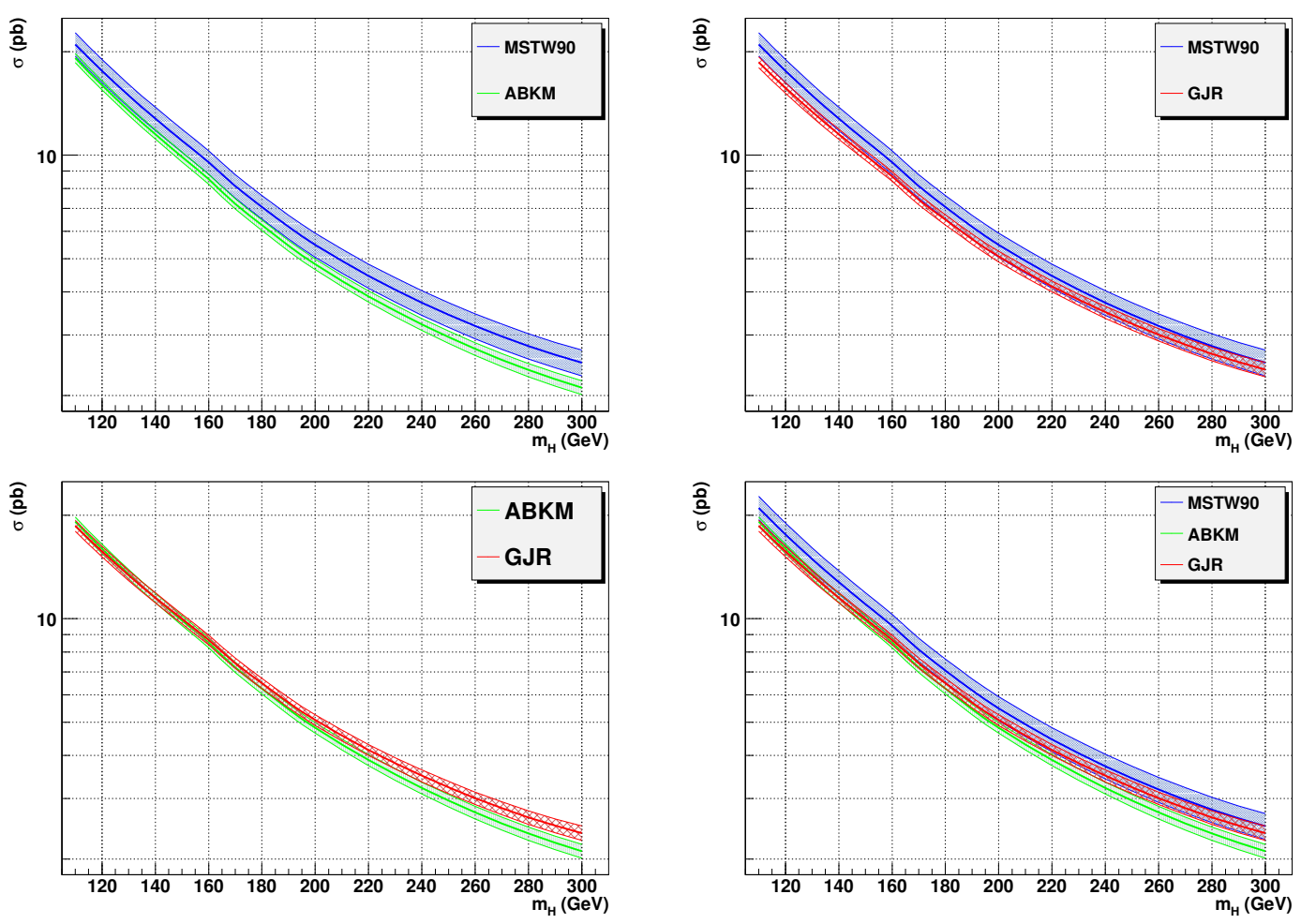

Figure 4. The Higgs production cross section at NNLO with three different PDF sets and their $\mathrm{PDF}+\alpha_{s}$ uncertainty bands, using the MSTW 90\% CL grids.

\begin{tabular}{|c||c||c|c|c|}
\hline$m_{H}$ & $\Gamma_{H}$ & $\sigma^{\text {ZWA }}$ & $\sigma^{\text {DEF }}$ & $\sigma^{\text {SEY }}$ \\
\hline 120 & 0.0038 & 17.57 & 17.66 & 17.57 \\
\hline 165 & 0.2432 & 8.78 & 8.874 & 8.735 \\
\hline 200 & 1.43 & 5.45 & 5.566 & 5.390 \\
\hline 400 & 29.5 & 1.988 & 1.799 & 1.766 \\
\hline 600 & 122 & 0.287 & 0.2409 & 0.3819 \\
\hline 800 & 301 & 0.04708 & 0.03982 & 0.15683 \\
\hline
\end{tabular}

Table 1. Total cross section for LHC at $\sqrt{s}=7 \mathrm{TeV}$ with MSTW PDFs with a finite width in the two schemes, $\sigma^{\mathrm{DEF}, \mathrm{SEY}}$, and in the zero width approximation denoted by $\sigma^{\mathrm{ZWA}}$.

In figure 6 the inclusive Higgs cross section calculated within the ZWA and the two finite width schemes is shown, as a function of the Higgs mass. The width is calculated by interpolating over a detailed grid $^{8}$ constructed with HDECAY [26]. The cross sections ${ }^{9}$ are shown in table 1. We note that the three calculations deviate widely for Higgs masses larger than $300 \mathrm{GeV}$. The deviation between the ZWA and the finite width schemes is expected since for large Higgs masses the width of the Higgs boson is comparable to its mass. It is

\footnotetext{
${ }^{8}$ The precision of the interpolation is always better than $2 \cdot 10^{-5}$.

${ }^{9}$ We use here the MSTW PDF set. Similar behavior is observed when using the other two NNLO PDF sets.
} 


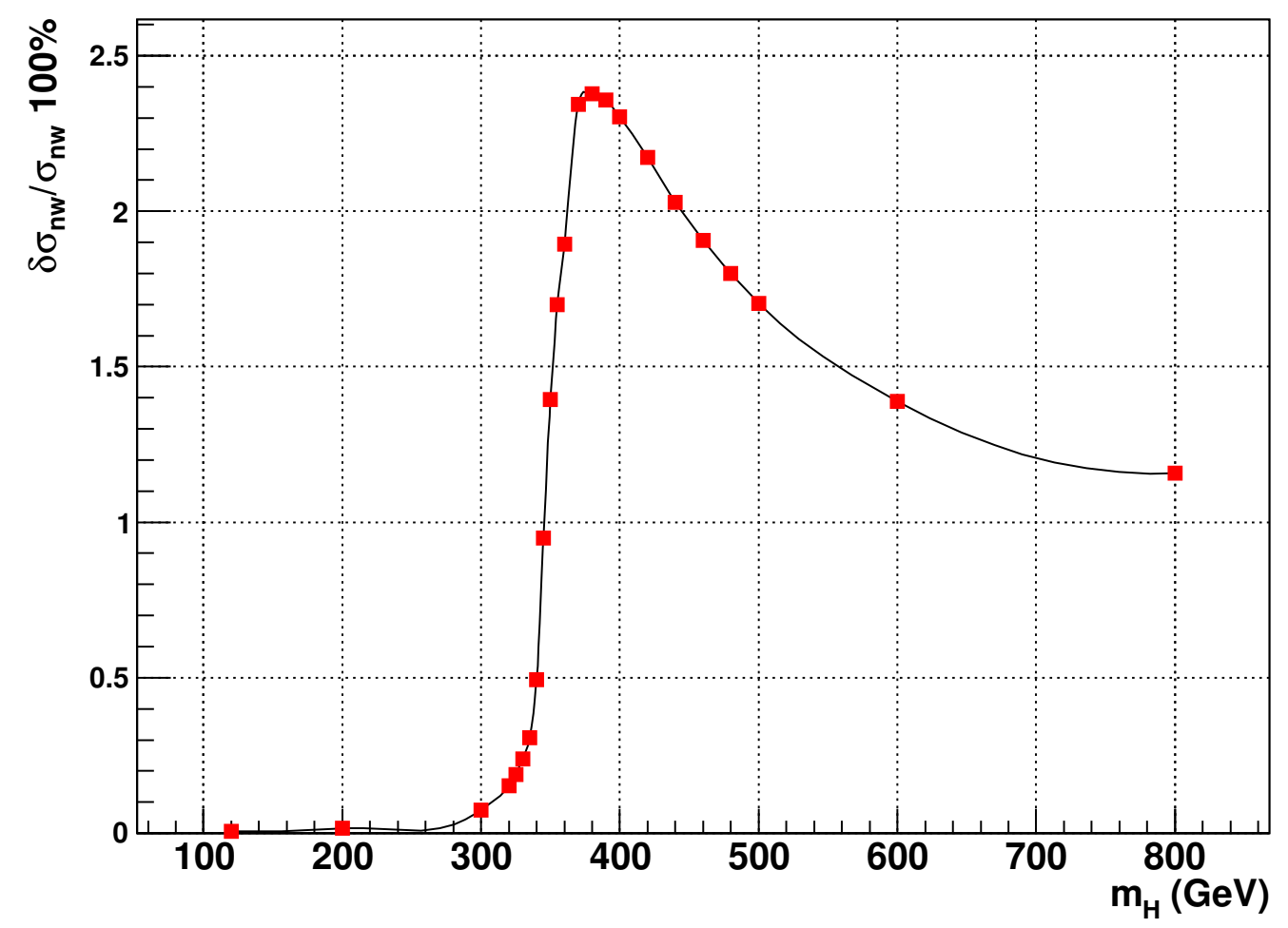

Figure 5. Relative difference $\delta \sigma_{n w} / \sigma_{n w}=\frac{\sigma-\sigma^{*}}{\sigma^{*}} \cdot 100 \%$ of the cross section for the top quark with a real mass, $\sigma^{*}$, and in the complex mass scheme with $\Gamma_{\text {top }}=2 \mathrm{GeV}$.

also evident that the finite width schemes deviate from each other in the high mass region, indicating a possibly large contribution due to signal and background interference which the Seymour scheme attempts to simulate.

Within this context, it is interesting to notice that the invariant mass distribution of the Higgs boson, shown in figure 7, gets significantly distorted in the high mass region, where the Higgs width is large. The distortion is spectacularly stronger in the case of the Seymour scheme, as a consequence of the fact that the scheme tries to simulate the effects of signal-background interference off the resonant peak. These effects become increasingly important for high Higgs masses.

In experimental searches for the Higgs boson where its invariant mass can be reconstructed from the momenta of the final state partons, as is the case for $H \rightarrow \gamma \gamma$, or $H \rightarrow Z Z$, it is beneficial for the analysis to impose a kinematical cut on the total invariant mass of the Higgs decay products. The invariant mass of the Higgs boson is then constrained in a window around the nominal Higgs boson mass, the size of which depends on the experimental resolution, see e.g. [72]. As a consequence, part of the signal is also cut.

The signal cross section that survives such kinematical cuts on the Higgs boson invariant mass can be calculated with iHixs and is shown in table 2 , for the window sizes employed in [72]. We observe that the reduction in the expected signal rate can reach $20 \%-40 \%$ for window choices smaller than the Higgs width. A non-negligible reduction of a few per cent persists even when the invariant mass window is larger than the nominal Higgs width, due to contributions from the tail of the Breit-Wigner distribution. 


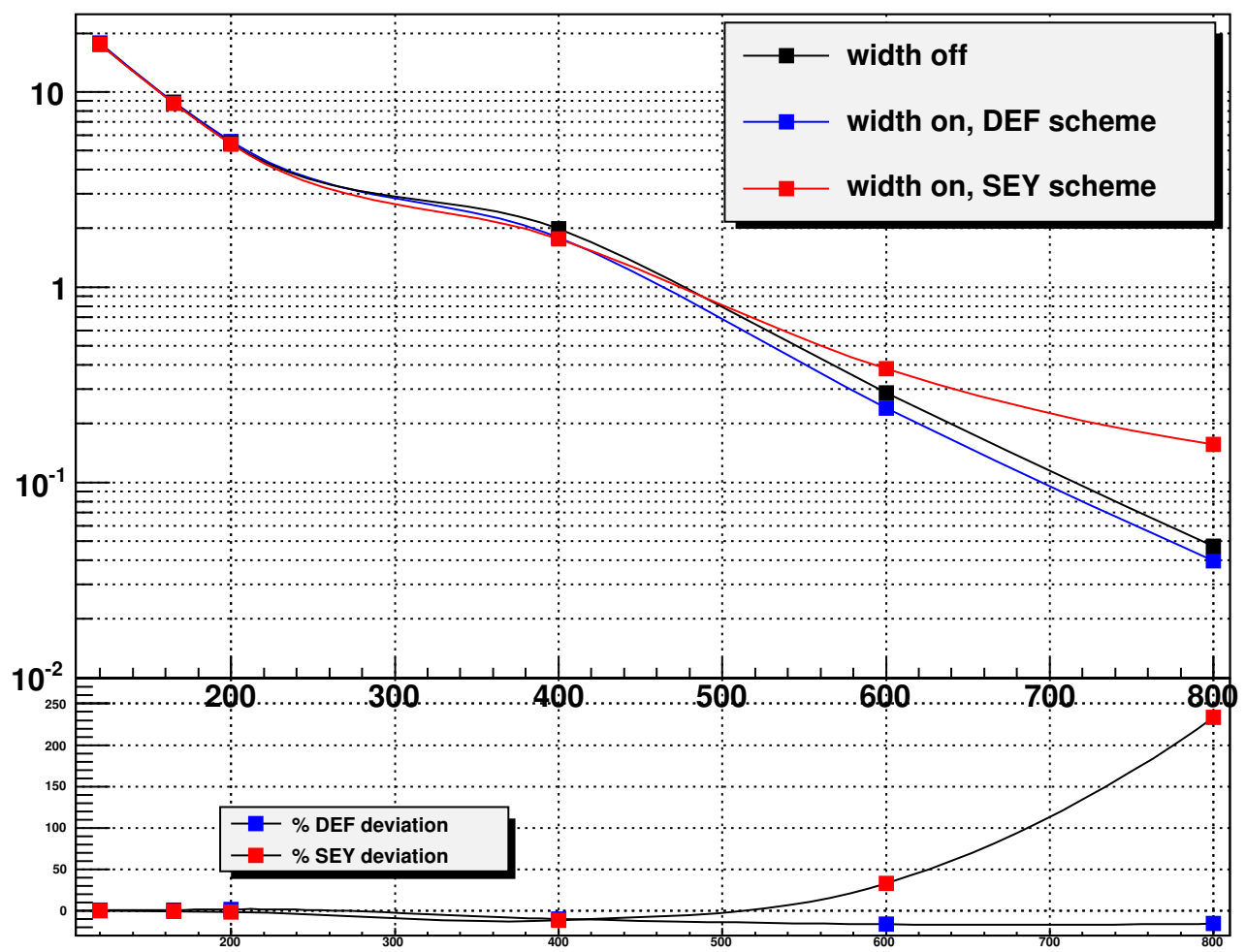

Figure 6. Comparison of the total cross section in the zero width approximation, $\sigma^{\text {ZWA }}$, with a finite width in the default scheme, $\sigma^{\mathrm{DEF}}$ and in the Seymour scheme, $\sigma^{\mathrm{SEY}}$. In the lower panel we show the relative error one makes when adopting the ZWA, defined as $\frac{\sigma-\sigma^{\mathrm{ZWA}}}{\sigma^{\mathrm{ZWA}}} \cdot 100 \%$.

\begin{tabular}{|c||c||c|c|c|c|c|}
\hline$m_{H}$ & $\Gamma_{H}$ & $\delta Q$ & $\sigma^{\text {DEF }}$ & $\sigma^{\text {DEF } ;}$ & $\sigma^{\mathrm{SEY}}$ & $\sigma^{\mathrm{SEY} ; w}$ \\
\hline 120 & 0.0038 & 5 & 17.66 & 17.56 & 17.57 & 17.56 \\
\hline 165 & 0.2432 & 5 & 8.874 & 8.62 & 8.735 & 8.62 \\
\hline 200 & 1.43 & 8 & 5.566 & 5.14 & 5.390 & 5.14 \\
\hline 400 & 29.5 & 34 & 1.799 & 1.448 & 1.766 & 1.447 \\
\hline 600 & 122 & 110 & 0.2409 & 0.1928 & 0.3819 & 0.2305 \\
\hline 800 & 301 & 300 & 0.03982 & 0.03451 & 0.15683 & 0.07510 \\
\hline
\end{tabular}

Table 2. Total cross section, $\sigma^{\mathrm{DEF}, \mathrm{SEY}}$ compared with the cross section in the invariant mass region $m_{H} \pm \delta Q$, denoted by $\sigma^{\mathrm{DEF} ; w}$ or $\sigma^{\mathrm{SEY} ;} w$, for LHC at $\sqrt{s}=7 \mathrm{TeV}$ with MSTW PDFs.

This effect can be estimated by parton shower Monte Carlo simulations which are the main simulation tools in experimental collaborations. It is important that a realistic line-shape for a heavy Higgs boson is implemented in these simulations. Common practice in experimental studies is to evaluate distributions with a LO or NLO Monte Carlo program interfaced with parton showers and then rescale the distributions by inclusive $\mathrm{K}$ factors. Those K-factors, however, depend on the scheme adopted for the Higgs width, as well as on the size of the experimental window, if one exists in the analysis, as shown in table 3 . 

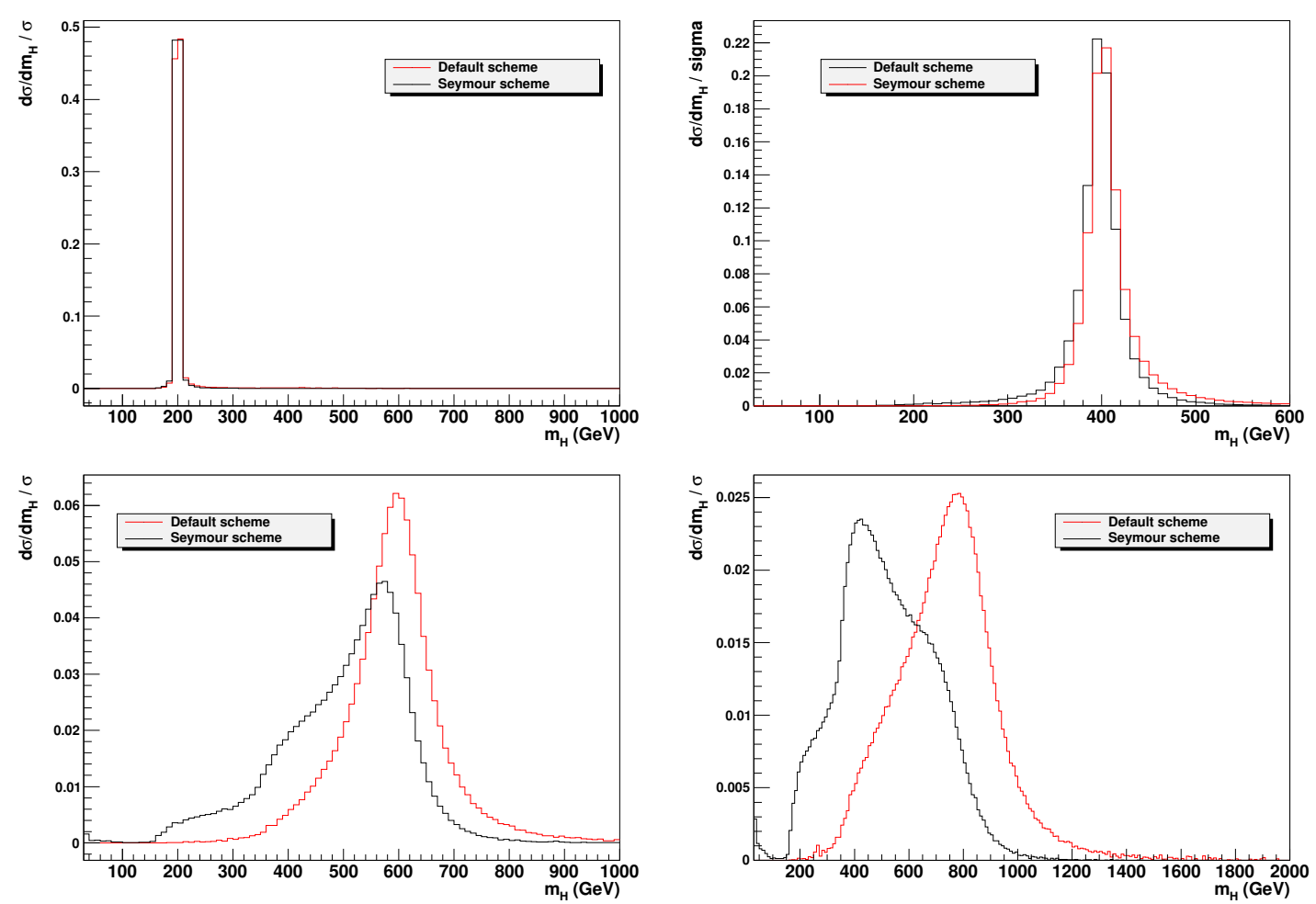

Figure 7. The invariant mass distribution of the Higgs boson with $m_{H}=200,400,600,800 \mathrm{GeV}$, in the default and the Seymour scheme.

\begin{tabular}{|c||c||c|c|c|c|c|c|}
\hline$m_{H}$ & $\Gamma_{H}$ & $\delta Q$ & $K_{\mathrm{NNLO}}^{\mathrm{DEF}}$ & $K_{\mathrm{NNLO}}^{\mathrm{DEF} ;}$ & $K_{\mathrm{NNLO}}^{\mathrm{SEY}}$ & $K_{\mathrm{NNLO}}^{\mathrm{SEY} ;}$ & $K_{\mathrm{NNLO}}^{\mathrm{ZWA}}$ \\
\hline 120 & 0.0038 & 5 & 2.05 & 2.05 & 2.05 & 2.05 & 2.05 \\
\hline 165 & 0.2432 & 5 & 2.02 & 2.03 & 2.016 & 2.04 & 2.033 \\
\hline 200 & 1.43 & 8 & 2.00 & 2.03 & 2.023 & 2.03 & 2.027 \\
\hline 400 & 29.5 & 34 & 1.90 & 1.94 & 1.94 & 1.95 & 1.95 \\
\hline 600 & 122 & 110 & 1.66 & 1.66 & 1.87 & 1.72 & 1.64 \\
\hline 800 & 301 & 300 & 1.63 & 1.59 & 2.07 & 1.77 & 1.54 \\
\hline
\end{tabular}

Table 3. NNLO K-factors with the width according to the Default scheme, $K_{\mathrm{NNLO}}^{\mathrm{DEF}}$, in the presence of kinematical windows, $K_{\mathrm{NNLO}}^{\mathrm{DEF} w}$, within the Seymour scheme, $K_{\mathrm{NNLO}}^{\mathrm{SEY}}$, and within the Seymour scheme in the presence of kinematical windows, $K_{\mathrm{NNLO}}^{\mathrm{SEY} ; w}$, compared with the K-factors in the zero width approximation, $K_{\mathrm{NNLO}}^{\mathrm{ZWA}}$.

When one departs from the zero width approximation, the branching ratios into the various final states also depend on the virtuality, as opposed to the nominal mass, of the Higgs boson. Assuming, at first, that all invariant masses are reconstructed experimentally, table 4 shows the difference between convoluting the branching ratio to a WW final state with the production cross section and the Breit-Wigner distribution, as in eq. (4.6), and just multiplying the total cross section with the branching ratio evaluated at the nominal Higgs mass value. We see that, in the low mass region, the relative deviations are very 


\begin{tabular}{|c||c||c|c|c|c|c|c|}
\hline$m_{H}$ & $\Gamma_{H}$ & $\mathrm{BR}_{H \rightarrow W W}$ & $\sigma_{p p \rightarrow H \rightarrow W W}$ & $\sigma_{1}$ & $\delta \sigma_{1} \%$ & $\sigma_{2}$ & $\delta \sigma_{2} \%$ \\
\hline 120 & 0.0038 & 0.1354 & 2.441 & 2.396 & -1.8 & 2.384 & -2.3 \\
\hline 165 & 0.2432 & 0.958 & 8.446 & 8.493 & 0.6 & 8.43 & -0.2 \\
\hline 200 & 1.43 & 0.742 & 4.123 & 4.132 & 0.2 & 4.05 & -1.7 \\
\hline 400 & 29.5 & 0.576 & 1.045 & 1.041 & -0.4 & 1.157 & 10.8 \\
\hline 600 & 122 & 0.560 & 0.132 & 0.131 & -0.8 & 0.163 & 23.8 \\
\hline 800 & 301 & 0.594 & 0.02269 & 0.02299 & 1.3 & 0.0285 & 25.6 \\
\hline
\end{tabular}

Table 4. Cross section convoluted with the branching ratio to WW, compared with the product $\sigma_{1}=\sigma_{p p \rightarrow H} \times \mathrm{BR}_{H \rightarrow W W}$, the relative deviation $\delta \sigma_{1}=\left(\frac{\sigma_{1}}{\sigma_{p p \rightarrow H \rightarrow W W}}-1\right) \cdot 100 \%$, and the product with the production cross section in the ZWA, $\sigma_{2}=\sigma_{p p \rightarrow H}^{\mathrm{ZWA}} \times \mathrm{BR}_{H \rightarrow W W}$, with the relative deviation $\delta \sigma_{2}=\left(\frac{\sigma_{2}}{\sigma_{p p \rightarrow H \rightarrow W W}}-1\right) \cdot 100 \%$. All numbers are for LHC at $\sqrt{s}=7 \mathrm{TeV}$ with MSTW PDFs.

small, at the per mille level, thanks to the stability of the branching ratio in the region sampled by the Breit-Wigner. In the high mass region they can become large, especially when off-resonant effects are taken into account, as is the case for the Seymour scheme.

An interesting theoretical question is whether the resummation recipe for the Higgs propagator employed in the vicinity of the Higgs resonance affects the tails of the invariant mass distribution, where we know that the correct propagator is the one appearing in eq. (4.4). To study this, we compare the invariant mass distribution with the distribution calculated in the region of the tails with the width of the Higgs boson set to zero in the denominator of the propagator. This distribution diverges at the peak, as expected, so we cut off a small region around the peak, of the order of $\Gamma\left(m_{H}\right) / 2$ or smaller, to assist convergence. The comparison is shown in figure 8 , for the default scheme and in figure 9 for the Seymour scheme. In both cases the tails of the distributions are described well, when $\left|Q-m_{H}\right|>\Gamma\left(m_{H}\right)$ which indicates that the precise prescription for the propagator resummation in the peak region doesn't affect the tails of the invariant mass distribution.

\subsection{Inclusive Higgs boson production in the presence of a fourth generation of quarks}

An extension of the Standard Model with an additional family of quark and leptons yields a large increase to the gluon fusion process. It is therefore tested more easily at the TEVATRON and the LHC than the SM scenario.

The most accurate computation of the total inclusive cross section for the production of the Higgs boson in a model with a fourth fermionic generation, for the LHC, has been presented recently in [69]. This calculation was performed in the ZWA.

The value of the physical Higgs width in such a scenario depends on the details of the model, and is generally bigger than the Standard Model Higgs width. In this paper, we would like to assess the impact of the width, making the rough assumption that its value is the same as in the SM width.

With this assumption, the relative difference between the production cross sections reported in [69] and a computation with the Higgs width on can reach the level of $2.5 \%$ in the high mass region, but is not significant for masses of $m_{H}<160 \mathrm{GeV}$, as shown in 

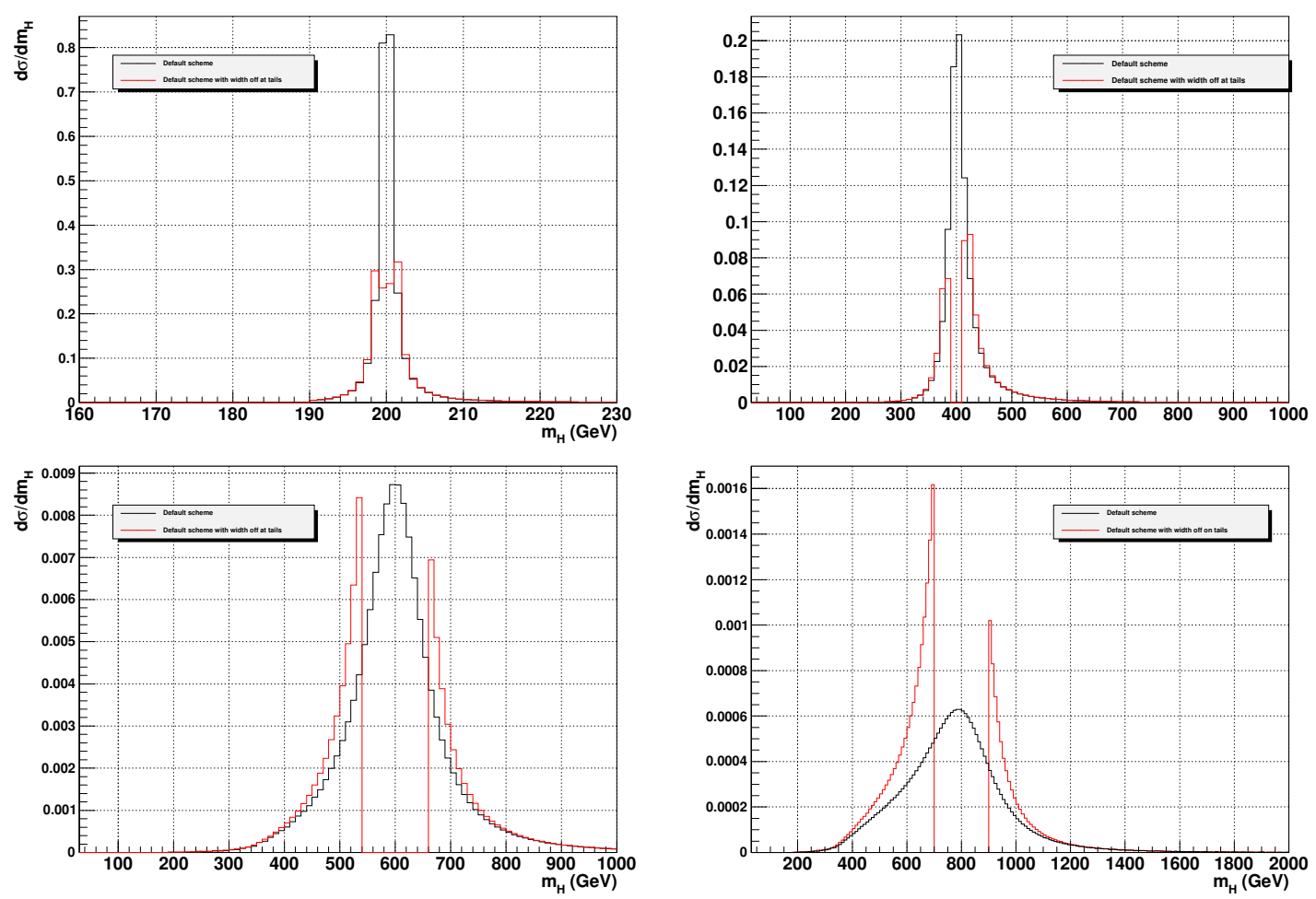

Figure 8. The invariant mass distribution of the Higgs boson with $m_{H}=200,400,600,800 \mathrm{GeV}$, in the default scheme, compared to the distribution of the tails computed with the off-resonant propagator.

figure 10. Let us remark, however, that in the high mass region the width of the Higgs boson is enhanced by the opening of new decay channels to third and fourth generation fermions, in a way that depends on the model.

Ref. [69] refrained from providing cross-sections for Higgs boson mass values higher than $m_{h}>300 \mathrm{GeV}$. Cross-section predictions in that range should always take into account finite width effects.

\section{The Higgs cross-section for a variable bottom-quark Yukawa interac- tion}

In the standard model the bottom-quark Yukawa coupling is much smaller than the topquark counterpartner,

$$
\frac{\lambda_{b}}{\lambda_{t}}=\frac{m_{b}}{m_{t}} \sim 0.02
$$

and the bottom-quark fusion cross section is about $2-3 \%$ of the gluon fusion cross section. This feature may however not be conserved in extensions of the standard model, which for example contain more than one Higgs field electroweak doublets. In such a scenario more than one physical Higgs boson arise after electroweak symmetry breaking. The Yukawa couplings may then be modified by further mixing angles of the model and can differ strongly from their Standard Model values. It is then possible that the bottom-quark 

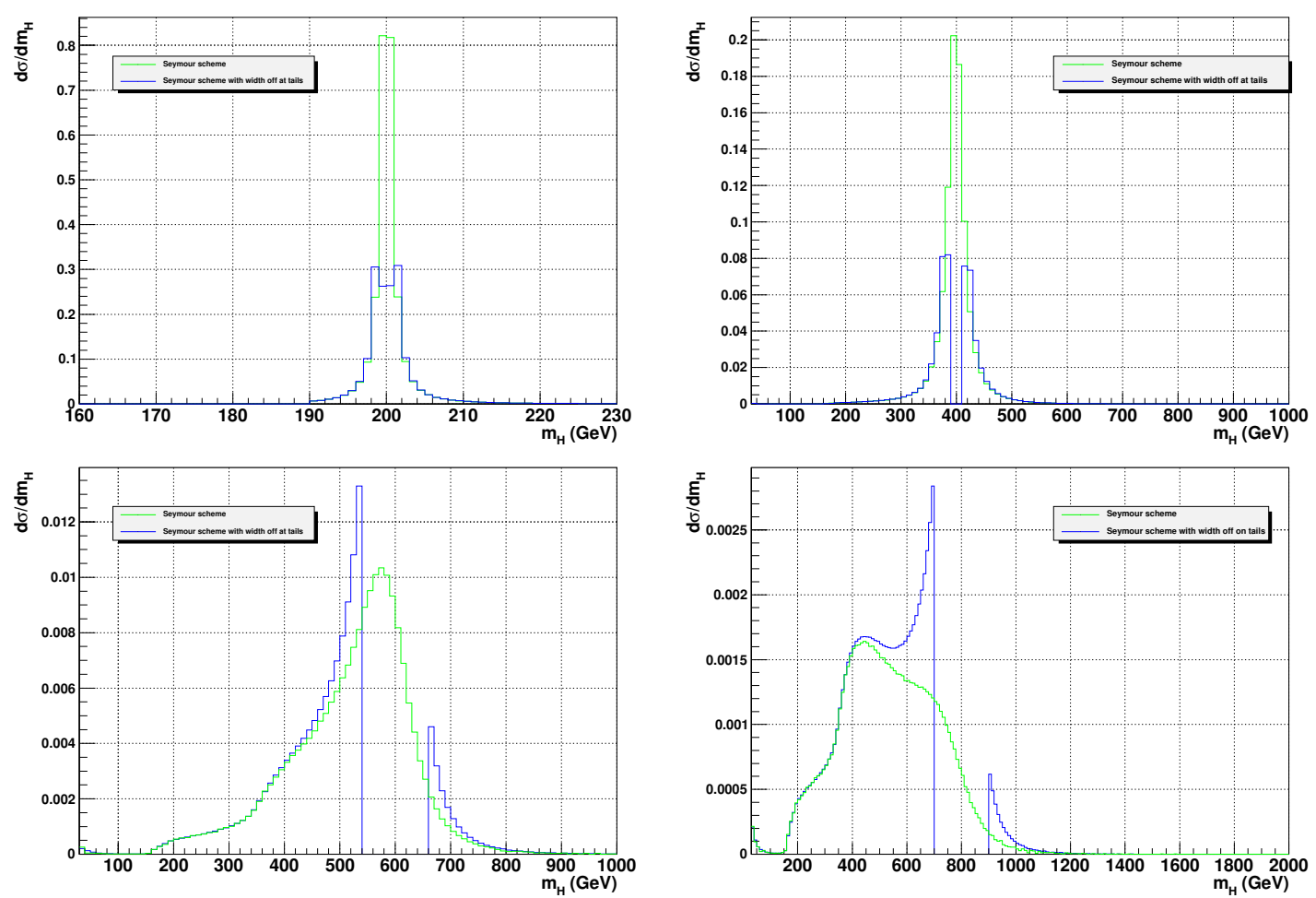

Figure 9. The invariant mass distribution of the Higgs boson with $m_{H}=200,400,600,800 \mathrm{GeV}$, in the Seymour scheme, compared to the distribution of the tails computed with the off-resonant propagator.

fusion and gluon fusion via bottom-quarks become very significant in comparison to gluon fusion via top-quark loops.

In this section, we would like to study the sum of the two processes, gluon fusion and bottom-quark fusion, which contribute to the inclusive production of a Higgs boson as a function of the bottom Yukawa coupling (we denote by $Y_{b}$ its value, normalized to the Standard Model).

In figure 11 we demonstrate the inclusive cross-section for gluon fusion and bottomquark fusion as well as their sum in the ZWA for a nominal Higgs mass of $120 \mathrm{GeV}$ at the LHC for $\sqrt{s}=7 \mathrm{TeV}$. We observe that for small $Y_{b}$ the cross-section is dominated by gluon fusion. For high $Y_{b}$, bottom-quark fusion is dominant but there is also a large contribution from gluon fusion, however via bottom rather than top-quarks. Notice that the gluon fusion cross-section reduces for moderate values of $Y_{b}$ due to a negative interference effect of top and bottom-quark loops.

It is often the practice that uncertainties for beyond the Standard Model Higgs bosons are taken over from studies within the Standard Model. This may not be a very bad option if new physics only introduces new heavy particles and does not alter Higgs couplings to light quarks significantly, since all such scenaria can be well described by a common effective theory operator. In our scenario however, we need to be more attentive. Our calculation of the gluon fusion cross section is exact through NLO for both bottom and top-quark 


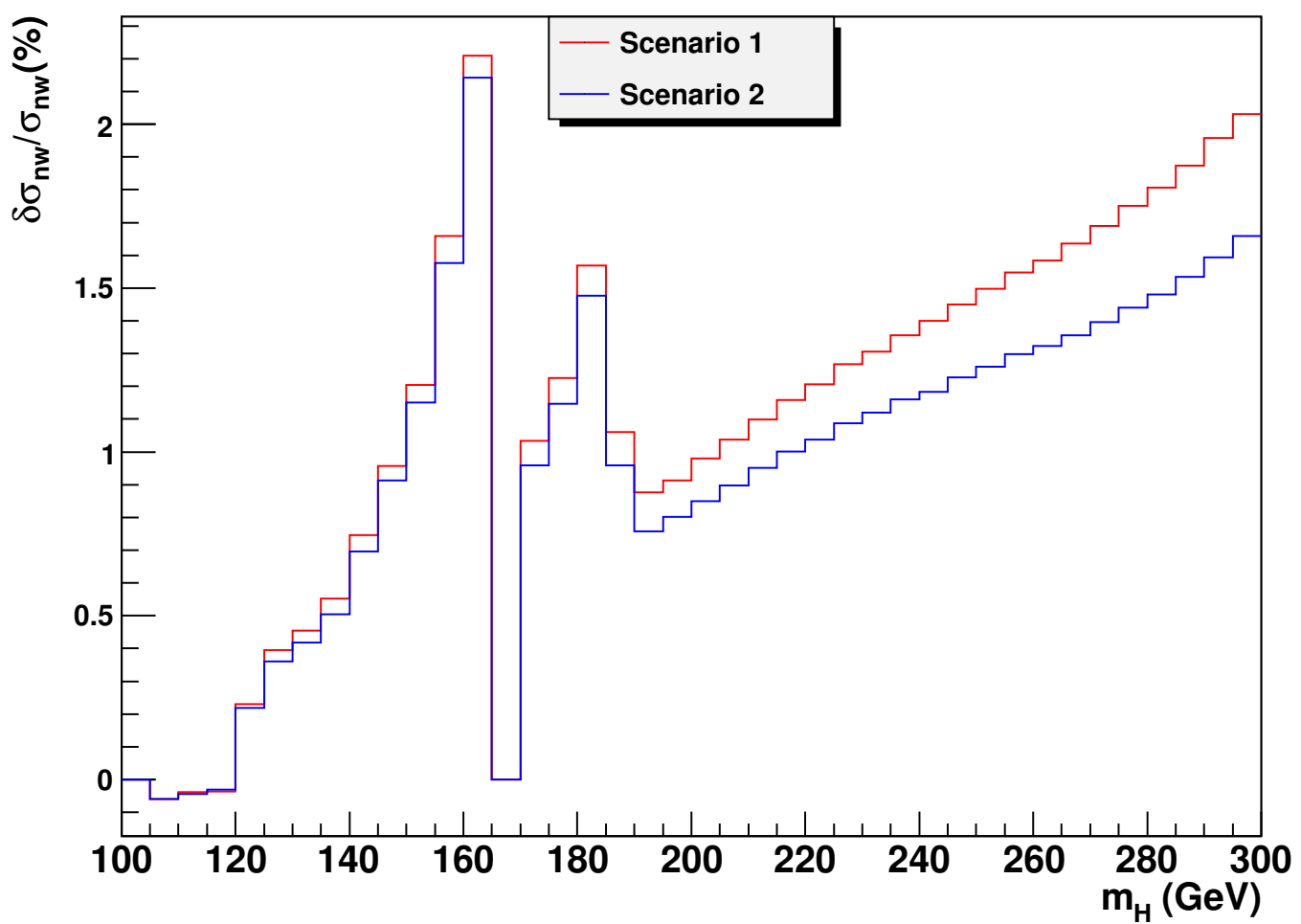

Figure 10. Relative difference $\frac{\sigma-\sigma^{\mathrm{ZWA}}}{\sigma^{\mathrm{ZWA}}} \cdot 100 \%$ of the cross section in SM4, within the two scenaria of [69], in the zero width approximation, $\sigma^{\mathrm{ZWA}}$, and in the approximation where $\Gamma_{\mathrm{SM} 4}\left(m_{h}\right)=$ $\Gamma_{\mathrm{SM}}\left(m_{H}\right)$. Scenario 1 refers to a fourth generation down quark of mass $m_{d_{4}}=300 \mathrm{GeV}$ while scenario 2 assumes $m_{d_{4}}=300 \mathrm{GeV}$. In both cases the fourth generation up quark has a mass that is determined by eq. (2) in section 3 of [69].

loop contributions. Our NNLO calculation includes only top-quark loops in the framework of HQET. For large $Y_{b}$ where bottom-quark loops dominate, our evaluation of the gluon fusion cross-section is reduced to NLO accuracy (not NNLO).

In figure 12 we demonstrate the scale variation of the cross-sections for gluon fusion, quark-bottom fusion and their sum, where we have combined scale uncertainties linearly. The scale uncertainty for gluon fusion increases for large $Y_{b}$ as expected due to the dominance of the NLO only bottom-quark corrections. The scale uncertainty of the inclusive cross-section is dominated by the largest cross-section contribution.

A large bottom-quark Yukawa coupling enhances the $H \rightarrow b \bar{b}$ decay width. The total width of the Higgs boson can be derived from the Standard Model total width and branching ratios as:

$$
\Gamma_{H}(Q)=\Gamma_{H}^{\mathrm{SM}}(Q) \times\left[\left(Y_{b}^{2}-1\right) \mathrm{Br}_{H \rightarrow b \bar{b}}^{\mathrm{SM}}(Q)+1\right] .
$$

In figure 13, we have plotted the combined bottom and gluon fusion cross section for a Higgs boson of $m_{H}=120 \mathrm{GeV}$ for two different invariant mass windows. While the width effects are small up to $Y_{b} \sim 30$, they significantly change the total cross section for higher $y_{b}$ values, due to the steep increase of the Higgs width as the mass crosses the vector boson thresholds. 


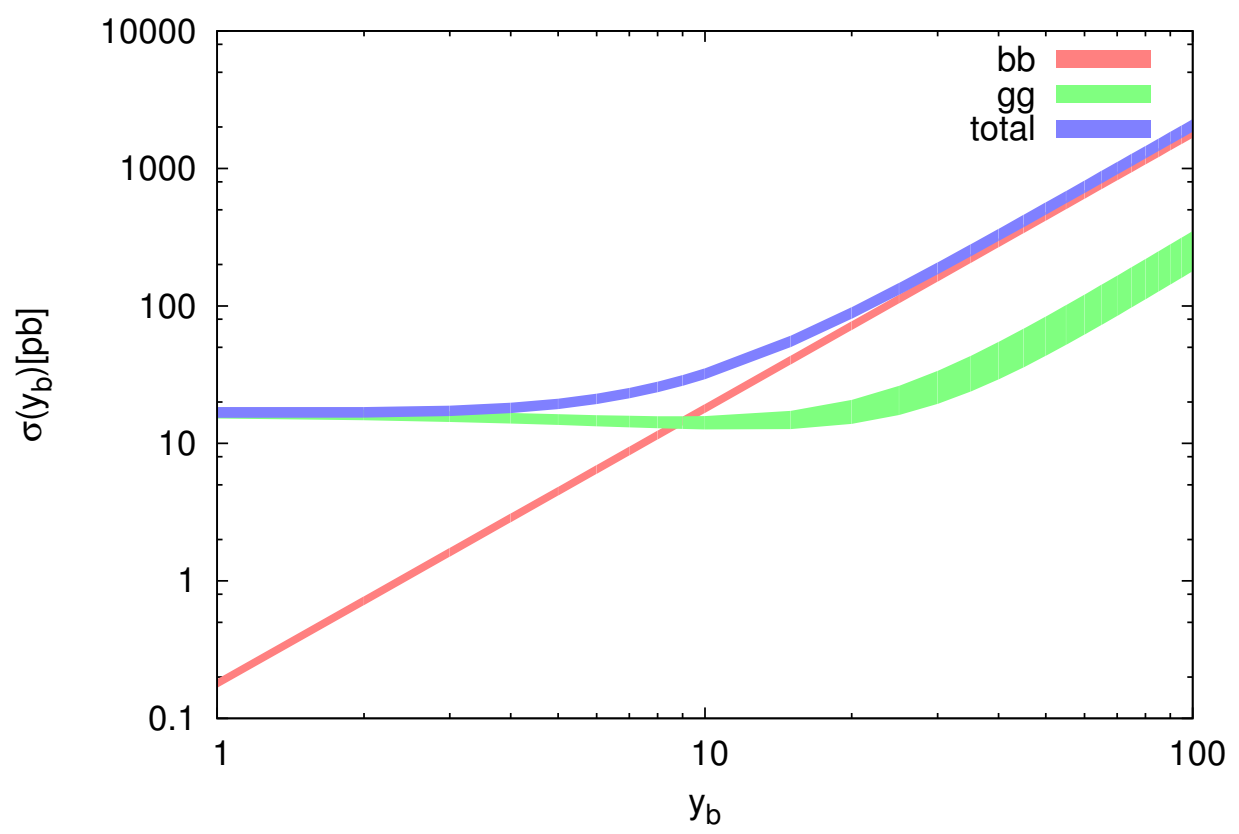

Figure 11. Single Higgs production cross sections as a function of the re-scaling factor of the bottom-quark Yukawa coupling $Y_{b}$. The bands represent the uncertainty due to the choice of factorization and renormalization scale.

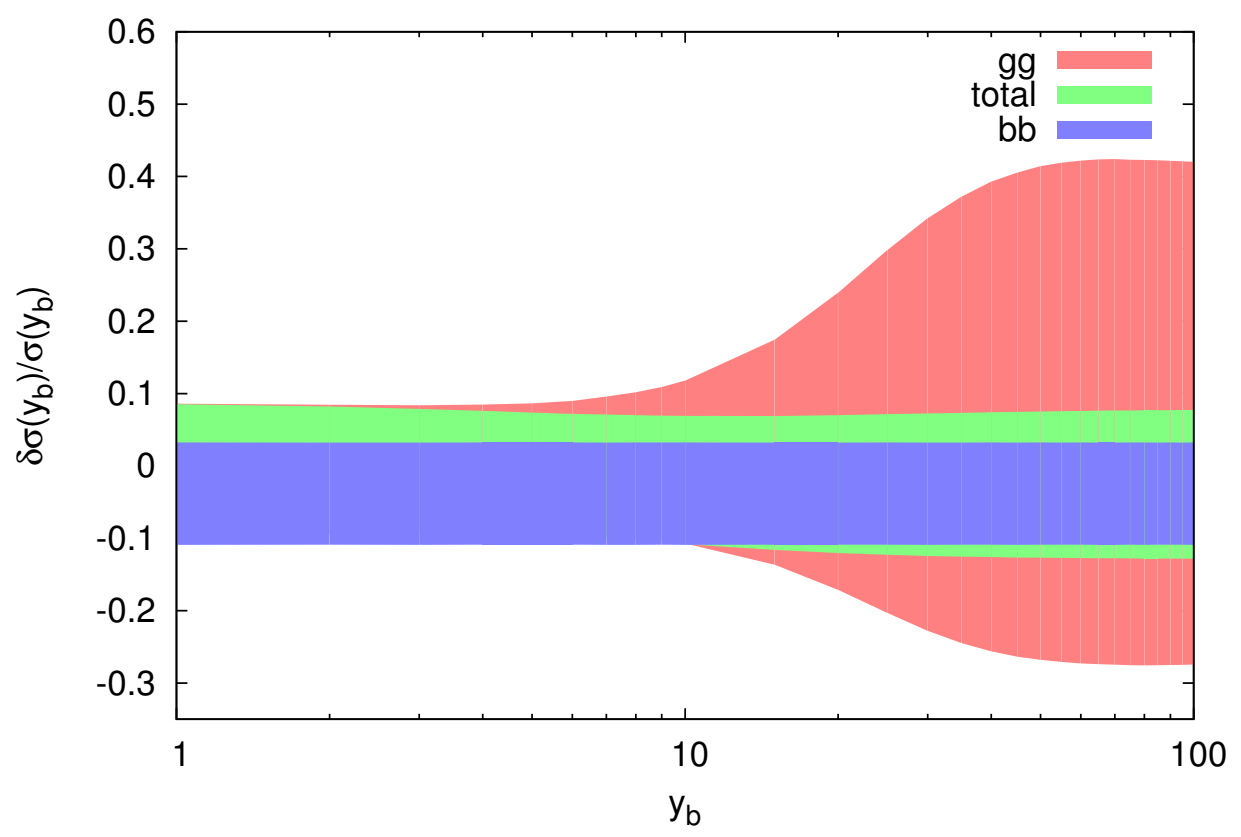

Figure 12. Relative scale uncertainties of single Higgs production cross sections as a function of $y_{b}$. 


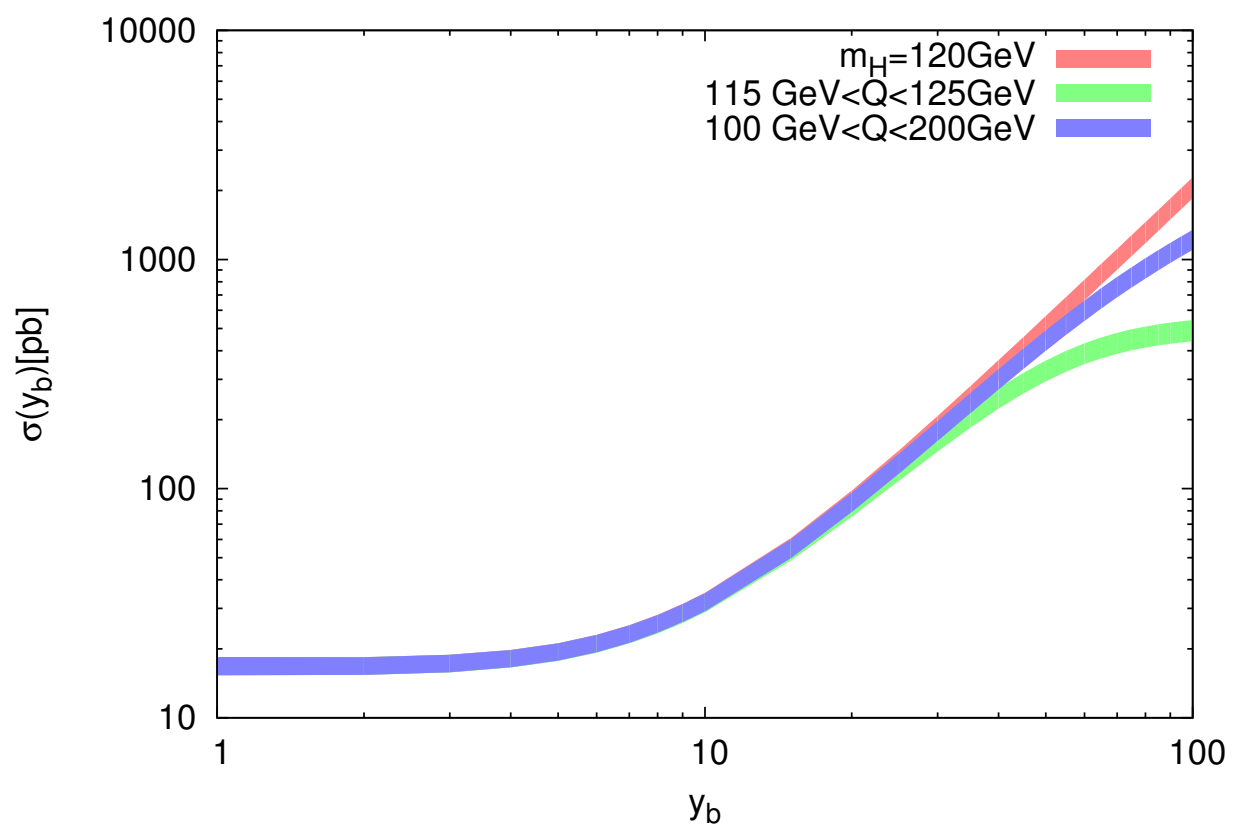

Figure 13. Combined gluon and bottom fusion cross section as a function of $y_{b}$ in on-shell and off-shell scheme. The bands represent the scale uncertainty.

\section{The iHixs program}

The source code for iHixs can be downloaded from its website at

http://www.phys.ethz.ch/ pheno/ihixs

Installation instructions can be found in the website and in the README file supplied in the distribution. Here, we briefly describe the main functionality of the code.

\subsection{Usage}

The various features of iHixs are controlled by an input runcard, a text file that is edited by the user. To run with a given runcard as input type in the installation directory:

\section{./ihixs -i runcard_name -o output_filename}

When no runcard is given, the program runs on the default card (called 'runcard') in the installation directory. When no output filename is given, the program writes the output in runcard_name. out.

The output consists of the total cross sections per perturbative order in QCD, together with the corresponding Monte-Carlo errors achieved and the PDF errors. Those are set to zero if no PDF uncertainty is requested in the runcard. The input runcard is also appended. 


\subsection{Setting options and variables}

In the runcard anything after a hash symbol, '\#', is considered as a comment and is ignored. The following options are available:

- pdf_provider: sets the PDF grid used. The user can choose between MSTW08, ABKM09 and GJR09. Within the MSTW PDFs there is also the option to switch confidence level from $68 \%$ to $90 \%$ and to use the MSTW grids with the strong coupling constant varied by one standard deviation from the best fit value. The exact filenames of the available grids are stated in the default runcard.

- effective_theory_flag: set to 0 for the exact LO and NLO QCD effects and HQET approximation for NNLO. Set to 1 for the improved HQET approximation through LO, NLO, NNLO.

- no_error_flag: set to 0 to calculate with PDF uncertainty, set to 1 to calculate without PDF uncertainty.

- collider: set to 'LHC' or 'TEVATRON'.

- Etot: the total center of mass collider energy. This option is ignored if the collider chosen above is Tevatron.

- mhiggs: the nominal mass of the Higgs boson.

- higgs_width_scheme: set to 0 for the default finite width scheme. Set to 1 for the Seymour scheme. For a description of these schemes see section 4 .

- higgs_width_grid: $=$ the path $^{10}$ of the file with the grid for the width of the Higgs, and the branching ratios to $\gamma \gamma, W W, Z Z$ and $b \bar{b}$ as a function of $m_{H}$. If no path is set the default grid is used, HdecayGrid.dat, constructed with Hdecay v.3.532 [26] with arguments that can be read in the header of the file. If the user supplies a grid file of his own, operating requirements are that the maximum number of grid points cannot exceed 16200, that the first three lines of the file are reserved for comments (so they are not read) and that the format of each line is respected, i.e. that the data is given in the order $m_{H}, \Gamma_{H}, \mathrm{BR}_{\gamma \gamma}, \mathrm{BR}_{W W}, \mathrm{BR}_{Z Z}, \mathrm{BR}_{b \bar{b}}$.

- min_mh: setting a minimum in the invariant mass of the Higgs boson. This allows the user to study the total cross section in the presence of kinematical cuts.

- max_mh: setting a maximum in the invariant mass of the Higgs boson.

- bin_flag: set to 1 to produce files with the bin-integrated Higgs invariant mass. Set to 0 not to produce it. The data files produced contain the cross section per bin, with the bin size set to $1 \mathrm{GeV}$, from 30 to $2000 \mathrm{GeV}$ at LO, NLO and NNLO. The files are named 'masshisto $\$ m_{H}$. $\$$ order', so e.g. for $m_{H}=200 \mathrm{GeV}$ the NLO file will be 'masshisto200.1'.

\footnotetext{
${ }^{10}$ Absolute or relative to the run directory.
} 
- muf/mhiggs: the ratio of the factorization scale and the Higgs mass.

- mur/mhiggs: the ratio of the renormalization scale and the Higgs mass.

- DecayMode: set to no_width for the zero width approximation total cross section, to 'total' for finite width total cross section, or to the decay modes 'gamma gamma', 'ZZ', 'WW', 'b b-bar'.

- ProductionMode: set to 'gg' for gluon fusion or to 'bb' for bottom-quark fusion.

- K_ewk: this is a global rescaling factor for all electroweak corrections. Set to 0.0 to switch them off.

- K_ewk_real: set to 0.0 to switch the electroweak corrections to $H+j$ off.

- K_ewk_real_b: set to 0.0 to switch the electroweak corrections to $H+j$ that include diagrams with massive quarks or Higgs boson in the loop, off.

- m_top: the pole mass of the top-quark.

- Gamma_top: the width of the top-quark.

- Y_top: rescaling factor for the SM Yukawa coupling of the top. Note that this can be set to an arbitrarily small positive value, but not to 0.0 exactly.

- m_bot: the $\overline{\mathrm{MS}}$ mass of the bottom-quark at $10 \mathrm{GeV}$.

- Gamma_bot: the width of the bottom-quark.

- Y_bot: rescaling factor for the SM Yukawa coupling of the bottom-quark.

- heavy quark: optional extra quarks in the model. The argument of this option should be formatted as $m_{Q}: \Gamma_{Q}: Y_{Q}$ where $Y_{Q}$ is the rescaling factor from a SM-like Yukawa coupling $m_{Q} / v$. For example, adding an extra $300 \mathrm{GeV}$ quark with width $1.2 \mathrm{GeV}$ and a Yukawa coupling that is $5.7 \frac{m_{Q}}{v}$ the user should type:

'heavy quark $=300.0: 1.2: 5.7$ '

- $\mathrm{m} \_\mathrm{Z}$ : the mass of the $\mathrm{Z}$ boson.

- Gamma_Z: the width of the Z boson.

- m_W: the mass of the $\mathrm{W}$ boson.

- Gamma_W: the width of the W boson.

- epsrel: sets the relative Monte-Carlo integration error.

- epsabs: sets the absolute Monte-Carlo integration error.

- nstart: sets the number of points per Vegas iteration. 
- nincrease: set the number of points by which the number of points per iteration increases.

- mineval: set the minimum number of points before ending the Monte-Carlo integration.

- maxeval: set the maximum number of points after which the integration ends.

- adapt to central only: set to 0 to force Vegas to adapt to all integrand. Set to 1 to adapt to the central integrand only. This is useful when running with PDF errors. Then each member of the PDF grid is treated as a separate integral. Adapting to the central only assumes that the peak structures of all integrals is similar which is a good approximation, and saves some CPU time.

- vegas_verbose: set to 0 for silent Vegas output. Set to 2 to have information about each iteration printed in the standard output (the console).

\subsection{Libraries used}

The program uses the following libraries:

- The Cuba library [78], v.2.1, for numerical integration. We use the Vegas algorithm, that employs importance sampling for variance reduction. For details on integration related arguments see [78] or the manual included in the Cuba-2.1 directory. We distribute Cuba-2.1 and compile it from source.

- The LHAPDF library [79]. We assume the library is installed by the user. See ref. [22] for details on installation.

- The package OneLOop $[19,20]$ for the evaluation of one-loop scalar integrals with complex masses. We use the library for the evaluation of finite box and triangle master integrals with massive propagators, necessary for the electroweak corrections to $H+j$ with massive fermions in the loop. We have checked our implementation of all other integrals against both OneLOop and QCDIoop [21] at the limit of zero width for the massive propagators. We distribute OneLOop and compile it from source.

- The CHAPLIN package [18], for evaluating harmonic polylogarithms up to weight four for any complex argument. This package is also distributed.

\section{Conclusions}

In this article, we have presented a computer program, iHixs, for the inclusive cross-section of the Higgs boson in gluon fusion and bottom-quark fusion. iHixs provides the most precise predictions for the Higgs boson rate at hadron colliders in fixed order perturbation theory, including QCD corrections through NNLO and electroweak corrections for virtual and real radiative partonic processes. iHixs is interfaced with the LHAPDF library and 
allows the assessment of uncertainties due to the various determinations of the parton densities which are available in the library.

In a time in which Higgs boson searches are growing in intensity, iHixs provides a very flexible tool which can assist in this effort. iHixs provides inclusive cross-section predictions in and beyond the Standard Model, by allowing modifications of Yukawa and electroweak couplings as well as the introduction of new quarks with arbitrary Yukawa couplings and masses. In addition, one can readily introduce effective Higgs-gluon interactions which can account for further beyond the Standard Model effects. ${ }^{11}$

The phenomenology of the Higgs boson and its production rates have been described extensively in the literature (recent updates can be found in refs. [50, 73-75]). We dedicated a very short analysis to issues which have been studied earlier at length, such as the magnitude of perturbative corrections and the convergence of the perturbative series beyond NNLO in QCD [76].

We have noticed that in recent experimental studies [3] the zero width approximation which is used for an expected light Higgs boson in the Standard Model is also employed for Higgs bosons or Higgs bosons with a sizable decay width. In this publication, we discuss finite width effects on the cross-section due to resonant Higgs boson diagrams. We also employ a prescription to estimate the effect of the signal-background interference for high Higgs boson masses which can be dramatic.

We believe that a realistic description of the Higgs line shape is necessary in setting exclusion limits for the Higgs boson. We remark that the description of the line shape in parton shower Monte-Carlo generators can be very different (for a comparison see ref. [77]). We have also demonstrated that the magnitude of radiative corrections (K-factor) differs from expectations in the zero width approximation when a large range of virtualities for the Higgs boson is sampled.

iHixs allows the user to perform exhaustive studies of the Higgs boson cross-section at hadron colliders. We are looking forward to comparing iHixs predictions with LHC data.

\section{Acknowledgments}

We are grateful to Elisabetta Furlan and Claude Duhr for numerous communications concerning their parallel research on issues relevant to the development of iHixs. Special thanks to Fabian Stoeckli for detailed discussions on the line-shape of a heavy Higgs boson and sharing his insight from parton shower Monte-Carlo simulations, and to Andreas van Hameren for his assistance with OneLOop. Research supported by the Swiss National Foundation under contract SNF 200020-126632.

\footnotetext{
${ }^{11}$ We are grateful to Elisabetta Furlan for extensive testings and feedback on implementing complicated extensions of the Standard Model in iHixs.
} 


\section{A Tables of Higgs cross-sections}

\begin{tabular}{|c||c|c|c|c|c|}
\hline$m_{H}$ & $\sigma(p b)$ & $\% \delta_{\mathrm{PDF}}^{+}$ & $\% \delta_{\mathrm{PDF}}^{-}$ & $\% \delta_{\mu_{F}}^{-}$ & $\% \delta_{\mu_{F}}^{+}$ \\
\hline 110.0 & 21.04 & 4.05 & -3.1 & 8.95 & -9.6 \\
\hline 115.0 & 19.22 & 4.05 & -3.11 & 8.78 & -9.55 \\
\hline 120.0 & 17.7 & 4.05 & -3.11 & 8.63 & -9.5 \\
\hline 125.0 & 16.3 & 4.04 & -3.12 & 8.48 & -9.46 \\
\hline 130.0 & 15.04 & 4.04 & -3.12 & 8.35 & -9.42 \\
\hline 135.0 & 13.92 & 4.03 & -3.14 & 8.23 & -9.37 \\
\hline 140.0 & 12.93 & 4.04 & -3.15 & 8.12 & -9.34 \\
\hline 145.0 & 12.03 & 4.03 & -3.16 & 8.0 & -9.32 \\
\hline 150.0 & 11.22 & 4.04 & -3.17 & 7.89 & -9.28 \\
\hline 155.0 & 10.49 & 4.05 & -3.18 & 7.8 & -9.25 \\
\hline 160.0 & 9.77 & 4.04 & -3.2 & 7.7 & -9.22 \\
\hline 165.0 & 8.87 & 4.05 & -3.22 & 7.65 & -9.2 \\
\hline 170.0 & 8.23 & 4.05 & -3.24 & 7.58 & -9.17 \\
\hline 175.0 & 7.69 & 4.05 & -3.26 & 7.51 & -9.15 \\
\hline 180.0 & 7.2 & 4.06 & -3.28 & 7.43 & -9.13 \\
\hline 185.0 & 6.69 & 4.06 & -3.29 & 7.37 & -9.13 \\
\hline 190.0 & 6.26 & 4.07 & -3.31 & 7.31 & -9.12 \\
\hline 195.0 & 5.89 & 4.07 & -3.34 & 7.24 & -9.1 \\
\hline 200.0 & 5.57 & 4.07 & -3.36 & 7.19 & -9.06 \\
\hline 210.0 & 5.01 & 4.09 & -3.39 & 7.06 & -9.02 \\
\hline 220.0 & 4.54 & 4.1 & -3.44 & 6.92 & -8.99 \\
\hline 230.0 & 4.14 & 4.11 & -3.48 & 6.79 & -8.96 \\
\hline 240.0 & 3.8 & 4.12 & -3.53 & 6.68 & -8.91 \\
\hline 250.0 & 3.5 & 4.14 & -3.56 & 6.57 & -8.85 \\
\hline 260.0 & 3.25 & 4.13 & -3.6 & 6.44 & -8.84 \\
\hline 270.0 & 3.04 & 4.17 & -3.65 & 6.3 & -8.79 \\
\hline 280.0 & 2.85 & 4.18 & -3.69 & 6.18 & -8.74 \\
\hline 290.0 & 2.7 & 4.19 & -3.73 & 6.04 & -8.65 \\
\hline 300.0 & 2.57 & 4.21 & -3.78 & 5.89 & -8.58 \\
\hline & & & & & \\
\hline
\end{tabular}

Table 5. Total cross section for LHC at $\sqrt{s}=7 \mathrm{TeV}$ with MSTW PDF errors (corresponding to $68 \% \mathrm{CL})$. 


\begin{tabular}{|c||c|c|c|c|c|}
\hline$m_{H}$ & $\sigma(p b)$ & $\% \delta_{\mathrm{PDF}}^{+}$ & $\% \delta_{\mathrm{PDF}}^{-}$ & $\% \delta_{\mu_{F}}^{-}$ & $\% \delta_{\mu_{F}}^{+}$ \\
\hline 110.0 & 19.2 & 3.1 & -3.1 & 8.16 & -9.19 \\
\hline 115.0 & 17.51 & 3.1 & -3.1 & 8.02 & -9.13 \\
\hline 120.0 & 16.07 & 3.1 & -3.1 & 7.89 & -9.09 \\
\hline 125.0 & 14.76 & 3.1 & -3.1 & 7.77 & -9.06 \\
\hline 130.0 & 13.6 & 3.1 & -3.1 & 7.65 & -9.02 \\
\hline 135.0 & 12.55 & 3.2 & -3.2 & 7.55 & -8.99 \\
\hline 140.0 & 11.63 & 3.2 & -3.2 & 7.44 & -8.95 \\
\hline 145.0 & 10.8 & 3.2 & -3.2 & 7.33 & -8.94 \\
\hline 150.0 & 10.05 & 3.3 & -3.3 & 7.26 & -8.9 \\
\hline 155.0 & 9.37 & 3.3 & -3.3 & 7.17 & -8.88 \\
\hline 160.0 & 8.71 & 3.3 & -3.3 & 7.1 & -8.85 \\
\hline 165.0 & 7.89 & 3.4 & -3.4 & 7.05 & -8.83 \\
\hline 170.0 & 7.3 & 3.4 & -3.4 & 6.99 & -8.82 \\
\hline 175.0 & 6.81 & 3.4 & -3.4 & 6.93 & -8.79 \\
\hline 180.0 & 6.36 & 3.5 & -3.5 & 6.86 & -8.79 \\
\hline 185.0 & 5.9 & 3.5 & -3.5 & 6.82 & -8.79 \\
\hline 190.0 & 5.5 & 3.5 & -3.5 & 6.77 & -8.77 \\
\hline 195.0 & 5.17 & 3.6 & -3.6 & 6.72 & -8.74 \\
\hline 200.0 & 4.88 & 3.6 & -3.6 & 6.66 & -8.71 \\
\hline 210.0 & 4.37 & 3.7 & -3.7 & 6.54 & -8.7 \\
\hline 220.0 & 3.94 & 3.8 & -3.8 & 6.43 & -8.67 \\
\hline 230.0 & 3.58 & 3.9 & -3.9 & 6.34 & -8.62 \\
\hline 240.0 & 3.27 & 4.0 & -4.0 & 6.22 & -8.61 \\
\hline 250.0 & 3.0 & 4.1 & -4.1 & 6.13 & -8.57 \\
\hline 260.0 & 2.77 & 4.2 & -4.2 & 6.03 & -8.52 \\
\hline 270.0 & 2.58 & 4.3 & -4.3 & 5.94 & -8.45 \\
\hline 280.0 & 2.41 & 4.4 & -4.4 & 5.82 & -8.4 \\
\hline 290.0 & 2.27 & 4.5 & -4.5 & 5.7 & -8.35 \\
\hline 300.0 & 2.15 & 4.6 & -4.6 & 5.55 & -8.28 \\
\hline & & & & & \\
\hline 15.9 & & & \\
\hline
\end{tabular}

Table 6. Total cross section for LHC at $\sqrt{s}=7 \mathrm{TeV}$ with ABKM PDF errors (corresponding to $68 \% \mathrm{CL})$. 


\begin{tabular}{|c||c|c|c|c|c|}
\hline$m_{H}$ & $\sigma(p b)$ & $\% \delta_{\text {PDF }}^{+}$ & $\% \delta_{\text {PDF }}^{-}$ & $\% \delta_{\mu_{F}}^{-}$ & $\% \delta_{\mu_{F}}^{+}$ \\
\hline 110.0 & 18.66 & 3.6 & -3.6 & 7.87 & -8.63 \\
\hline 115.0 & 17.1 & 3.5 & -3.5 & 7.73 & -8.59 \\
\hline 120.0 & 15.79 & 3.5 & -3.5 & 7.58 & -8.55 \\
\hline 125.0 & 14.58 & 3.5 & -3.5 & 7.46 & -8.5 \\
\hline 130.0 & 13.49 & 3.4 & -3.4 & 7.35 & -8.46 \\
\hline 135.0 & 12.52 & 3.4 & -3.4 & 7.25 & -8.42 \\
\hline 140.0 & 11.66 & 3.4 & -3.4 & 7.14 & -8.39 \\
\hline 145.0 & 10.88 & 3.4 & -3.4 & 7.04 & -8.36 \\
\hline 150.0 & 10.17 & 3.3 & -3.3 & 6.95 & -8.33 \\
\hline 155.0 & 9.53 & 3.3 & 3.3 & 6.85 & 8.31 \\
\hline 160.0 & 8.89 & 3.3 & 3.3 & 6.77 & 8.3 \\
\hline 165.0 & 8.09 & 3.4 & 3.4 & 6.73 & 8.29 \\
\hline 170.0 & 7.53 & 3.4 & 3.4 & 6.66 & 8.31 \\
\hline 175.0 & 7.05 & 3.4 & 3.4 & 6.58 & 8.33 \\
\hline 180.0 & 6.62 & 3.4 & 3.4 & 6.53 & 8.36 \\
\hline 185.0 & 6.17 & 3.4 & 3.4 & 6.46 & 8.42 \\
\hline 190.0 & 5.78 & 3.5 & 3.5 & 6.4 & -8.46 \\
\hline 195.0 & 5.45 & 3.5 & 3.5 & 6.35 & 8.5 \\
\hline 200.0 & 5.16 & 3.6 & 3.6 & 6.29 & 8.54 \\
\hline 210.0 & 4.66 & 3.6 & 3.6 & 6.18 & 8.62 \\
\hline 220.0 & 4.24 & 3.7 & 3.7 & 6.04 & 8.7 \\
\hline 230.0 & 3.88 & 3.8 & 3.8 & 5.94 & 8.75 \\
\hline 240.0 & 3.57 & 4.0 & 4.0 & 5.83 & 8.81 \\
\hline 250.0 & 3.31 & 4.1 & 4.1 & 5.7 & -8.9 \\
\hline 260.0 & 3.08 & 4.2 & 4.2 & 5.61 & 8.92 \\
\hline 270.0 & 2.88 & 4.4 & 4.4 & 5.49 & 8.96 \\
\hline 280.0 & 2.72 & 4.5 & 4.5 & 5.38 & 8.97 \\
\hline 290.0 & 2.58 & 4.6 & 4.6 & 5.24 & 9.0 \\
\hline 300.0 & 2.46 & 4.8 & 4.8 & 5.09 & 9.01 \\
\hline
\end{tabular}

Table 7. Total cross section for LHC at $\sqrt{s}=7 \mathrm{TeV}$ with GJR PDF errors (corresponding to $68 \% \mathrm{CL})$. 


\section{B Matrix element coefficients}

\section{B.1 Master integral definitions}

In the following we will use the shorthand notation

$$
p_{i_{1} i_{2} \ldots i_{n}}=p_{i_{1}}+p_{i_{2}}+\ldots+p_{i_{n}}
$$

and define the Mandelstam variables

$$
s=\left(p_{12}\right)^{2}, \quad t=\left(p_{23}\right)^{2}, \quad u=\left(p_{13}\right)^{2}, \quad m_{H}^{2}=\left(p_{123}\right)^{2}
$$

where $p_{1}^{2}=p_{2}^{2}=p_{3}^{2}=0$. The master integrals are then given by

$$
\begin{aligned}
& \operatorname{Tadp}\left(m^{2}\right)=\int \frac{d^{D} k}{i \pi^{D / 2}} \frac{1}{\left[k^{2}-m^{2}\right]} \\
& \operatorname{Bub}\left(s, m^{2}\right)=\int \frac{d^{D} k}{i \pi^{D / 2}} \frac{1}{\left[k^{2}-m^{2}\right]\left[\left(k+p_{12}\right)^{2}-m^{2}\right]} \\
& \operatorname{Tria}\left(s, m^{2}\right)=s \cdot \int \frac{d^{D} k}{i \pi^{D / 2}} \frac{1}{\left[k^{2}-m^{2}\right]\left[\left(k+p_{1}\right)^{2}-m^{2}\right]\left[\left(k+p_{12}\right)^{2}-m^{2}\right]} \\
& \operatorname{Box}\left(s, t, u, m^{2}\right)= \\
& s \cdot t \cdot \int \frac{d^{D} k}{i \pi^{D / 2}} \frac{1}{\left[k^{2}-m^{2}\right]\left[\left(k+p_{1}\right)^{2}-m^{2}\right]\left[\left(k+p_{12}\right)^{2}-m^{2}\right]\left[\left(k+p_{123}\right)^{2}-m^{2}\right]} \\
& \operatorname{BubE}\left(s, m_{1}^{2}, m_{2}^{2}\right)=\int \frac{d^{D} k}{i \pi^{D / 2}} \frac{1}{\left[k^{2}-m_{1}^{2}\right]\left[\left(k+p_{12}\right)^{2}-m_{2}^{2}\right]} \\
& \operatorname{TriaE}\left(s, m_{H}^{2}, m_{1}^{2}, m_{2}^{2}\right)=s \cdot \int \frac{d^{D} k}{i \pi^{D / 2}} \frac{1}{\left[k^{2}-m_{1}^{2}\right]\left[\left(k+p_{12}\right)^{2}-m_{2}^{2}\right]\left[\left(k+p_{123}\right)^{2}-m_{1}^{2}\right]} \\
& \operatorname{TriaF}\left(s, m_{1}^{2}, m_{2}^{2}\right)=s \cdot \int \frac{d^{D} k}{i \pi^{D / 2}} \frac{1}{\left[k^{2}-m_{1}^{2}\right]\left[\left(k+p_{1}\right)^{2}-m_{2}^{2}\right]\left[\left(k+p_{12}\right)^{2}-m_{2}^{2}\right]} \\
& s \cdot t \cdot \int \frac{d^{D} k}{i \pi^{D / 2}} \frac{1}{\left[k^{2}-m_{1}^{2}\right]\left[\left(k+p_{1}\right)^{2}-m_{2}^{2}\right]\left[\left(k+p_{12}\right)^{2}-m_{2}^{2}\right]\left[\left(k+p_{123}\right)^{2}-m_{1}^{2}\right]} . \\
& \operatorname{BubD}\left(s, m_{q}^{2}, t\right)=\operatorname{Bub}\left(s, m_{q}^{2}\right)-\operatorname{Bub}\left(t, m_{q}^{2}\right) . \\
& A_{g g g H}^{1 q}\left(s, t, u, m_{q}^{2}\right)=2 \frac{(2 s t+t u+s u) \operatorname{Box}\left(s, t, u, m_{q}^{2}\right)}{u}+2 \frac{(2 t u+s t+s u) \operatorname{Box}\left(t, u, s, m_{q}^{2}\right)}{s} \\
& +2 \frac{(2 s u+t u+s t) \operatorname{Box}\left(u, s, t, m_{q}^{2}\right)}{t}-8 \frac{\left(4 t u+t^{2}+u^{2}\right) s \operatorname{BubD}\left(s, m_{q}^{2}, s+t+u\right)}{(u+t)^{2}} \\
& -8 \frac{\left(u^{2}+4 s u+s^{2}\right) t \operatorname{BubD}\left(t, m_{q}^{2}, s+t+u\right)}{(u+s)^{2}}-8 \frac{\left(4 s t+s^{2}+t^{2}\right) u \operatorname{BubD}\left(u, m_{q}^{2}, s+t+u\right)}{(t+s)^{2}} \\
& +\left(4 \frac{5 s^{4} u t^{2}+5 s u^{4} t^{2}+8 s u^{3} t^{3}+10 s^{2} u^{3} t^{2}+10 s^{2} u^{2} t^{3}+10 s^{3} u^{2} t^{2}+8 s^{3} u t^{3}+5 t^{4} u^{2} s}{t(u+t) s(u+s) u(t+s)}\right.
\end{aligned}
$$




$$
\begin{aligned}
& +\frac{5 s^{2} t^{4} u+5 t s^{2} u^{4}+8 t s^{3} u^{3}+5 s^{4} u^{2} t+2 u^{4} t^{3}+2 u^{3} t^{4}+2 s^{3} u^{4}+2 s^{4} u^{3}+2 s^{3} t^{4}+2 s^{4} t^{3}}{t(u+t) s(u+s) u(t+s)} \\
& -16 m_{q}^{2}\left[\frac{8 t^{3} u^{2} s+4 t u^{4} s+8 t u^{3} s^{2}+8 s^{2} t^{3} u+8 t^{2} u^{3} s+4 t^{4} s u+4 t^{3} u^{3}+3 t^{2} u^{4}+3 t^{4} s^{2}}{(u+s)^{2}(t+s)^{2}(u+t)^{2}}\right. \\
& \left.\left.+\frac{3 s^{2} u^{4}+3 t^{4} u^{2}+4 s^{3} u^{3}+3 s^{4} u^{2}+3 s^{4} t^{2}+4 t^{3} s^{3}+8 s^{3} u^{2} t+4 s^{4} u t+6 s^{2} t^{2} u^{2}+8 s^{3} t^{2} u}{(u+s)^{2}(t+s)^{2}(u+t)^{2}}\right]\right) \\
& \times \operatorname{Tria}\left(s+t+u, m_{q}^{2}\right) \\
& +\left(16 \frac{\left(u^{2}+t^{2}\right) m_{q}^{2}}{(u+t)^{2}}-4 \frac{2 t^{3} s+3 s t^{2} u+3 s u^{2} t+2 s u^{3}+t^{3} u+u^{3} t}{t u(u+t)}\right) \operatorname{Tria}\left(s, m_{q}^{2}\right) \\
& +\left(16 \frac{\left(s^{2}+u^{2}\right) m_{q}^{2}}{(u+s)^{2}}-4 \frac{2 t s^{3}+3 s^{2} u t+3 s u^{2} t+2 u^{3} t+s u^{3}+s^{3} u}{s u(u+s)}\right) \operatorname{Tria}\left(t, m_{q}^{2}\right) \\
& +\left(16 \frac{\left(t^{2}+s^{2}\right) m_{q}^{2}}{(t+s)^{2}}-4 \frac{2 s^{3} u+3 s^{2} u t+3 s t^{2} u+2 t^{3} u+t^{3} s+t s^{3}}{s t(t+s)}\right) \operatorname{Tria}\left(u, m_{q}^{2}\right) \\
& -16 \frac{s t^{2} u+s^{2} u t+s u^{2} t+s^{2} t^{2}+t^{3} u+s^{3} u+t^{3} s+t^{2} u^{2}+s u^{3}+t s^{3}+u^{3} t+s^{2} u^{2}}{(u+t)(u+s)(t+s)} \\
& A_{g g g H}^{2 q}\left(s, t, u, m_{q}^{2}\right)=\left(-16 m_{q}^{2}-2 \frac{-u^{2}-t u+2 s t}{u}\right) \operatorname{Box}\left(s, t, u, m_{q}^{2}\right) \\
& +\left(-16 m_{q}^{2}+2 \frac{s t+s^{2}-2 t u}{s}\right) \operatorname{Box}\left(t, u, s, m_{q}^{2}\right) \\
& +\left(16 m_{q}^{2}+4 \frac{s u}{t}\right) \operatorname{Box}\left(u, s, t, m_{q}^{2}\right)+8 \frac{s(-u+t) \operatorname{BubD}\left(s, m_{q}^{2}, s+t+u\right)}{u+t} \\
& +8 \frac{\left(u^{2}+4 s u+s^{2}\right) t \operatorname{BubD}\left(t, m_{q}^{2}, s+t+u\right)}{(u+s)^{2}}-8 \frac{(s-t) u \operatorname{BubD}\left(u, m_{q}^{2}, s+t+u\right)}{t+s} \\
& +\left(-16 \frac{\left(u^{3} t+3 t^{2} u^{2}+5 s^{2} u t+3 s^{2} t^{2}-s u^{3}+5 s u^{2} t+8 s t^{2} u-s^{3} u+t s^{3}\right) m_{q}^{2}}{(u+s)^{2}(t+s)(u+t)}\right. \\
& \left.-4 \frac{2 s^{3} t^{2}-s t^{2} u^{2}+2 t^{2} u^{3}-s^{2} t^{2} u-2 t u^{2} s^{2}-2 s^{2} u^{3}-2 s^{3} u^{2}}{(u+s) s t u}\right) \operatorname{Tria}\left(s+t+u, m_{q}^{2}\right) \\
& +\left(16 \frac{(-u+t) m_{q}^{2}}{u+t}+4 \frac{2 t^{2} s-t^{2} u-2 u^{2} s}{t u}\right) \operatorname{Tria}\left(s, m_{q}^{2}\right) \\
& +\left(16 \frac{\left(u^{2}+4 s u+s^{2}\right) m_{q}^{2}}{(u+s)^{2}}+4 \frac{2 t s^{3}-2 s^{2} u^{2}+s^{2} u t+s u^{2} t+2 u^{3} t}{s u(u+s)}\right) \operatorname{Tria}\left(t, m_{q}^{2}\right) \\
& +\left(-16 \frac{(s-t) m_{q}^{2}}{t+s}-4 \frac{2 s^{2} u+t^{2} s-2 t^{2} u}{s t}\right) \operatorname{Tria}\left(u, m_{q}^{2}\right)-16 \frac{s u+s t+t u}{u+s} \\
& A_{g g g H}^{3 q}\left(s, t, u, m_{q}^{2}\right)=A_{2}\left(t, u, s, m_{q}^{2}\right) \\
& A_{g g g H}^{4 q}\left(s, t, u, m_{q}^{2}\right)=A_{2}\left(u, s, t, m_{q}^{2}\right)
\end{aligned}
$$




\section{B.3 $A_{q \bar{q} g H}$}

Defining

$$
\tau_{q} \equiv \frac{4 m_{q}^{2}}{m_{H}^{2}}
$$

we have

$$
\begin{aligned}
\mathrm{A}_{q q g H}\left(\tau_{q}, y, m_{H}^{2}\right)=-\frac{3}{4}\left\{\frac{-2 A\left(\tau_{q}\right)}{1-y}\right. & \\
+ & \frac{2 y}{(1-y)^{2}} \operatorname{BubD}\left(y \cdot m_{H}^{2}, m_{q}^{2}, m_{H}^{2}\right)+\frac{\tau y}{(1-y)^{2}}\left[\frac{\operatorname{Tria}\left(y \cdot m_{H}^{2}, m_{q}^{2}\right)}{y}-\operatorname{Tria}\left(m_{H}^{2}, m_{q}^{2}\right)\right] \\
& \left.-\frac{1}{1-y}(1-\epsilon) \operatorname{Tria}\left(y \cdot m_{H}^{2}, m_{q}^{2}\right)\right\}
\end{aligned}
$$

Notice that for $y_{12} \rightarrow 0$ we have that

$$
\lim _{y_{12} \rightarrow 0} \mathrm{~A}_{q q g}\left(\tau_{q}, y_{12}, m_{H}^{2}\right)=A\left(\tau_{q}, m_{H}^{2}\right)
$$

which is the familiar kernel of the born $g g \rightarrow h$ amplitude.

\section{B.4 $A_{\text {ewk }}$}

$$
\begin{aligned}
A_{\mathrm{ewk}}\left(s, t, u, m_{H}, m_{z}\right)= & \frac{t\left(m_{z}^{2}-s\right)}{s\left[m_{z}^{2}(s+t)-s t\right]} \operatorname{BoxE}^{(d=6)}\left(s, t, m_{H}^{2}, m_{z}^{2}, 0\right) \\
& +\frac{m_{z}^{2}\left(m_{z}^{2}-s\right)}{s\left[m_{z}^{2}(s+t)-s t\right]} \operatorname{TriaE}\left(s, m_{H}^{2}, m_{z}^{2}, 0\right) \\
& +\frac{1}{s}\left[1-\frac{m_{z}^{2}}{s+u}-\frac{m_{z}^{4}}{m_{z}^{2}(s+t)-s t}\right] \operatorname{TriaE}\left(t, m_{H}^{2}, m_{z}^{2}, 0\right) \\
& +\frac{\operatorname{Bub}\left(m_{H}^{2}, m_{z}^{2}\right)-\operatorname{BubE}\left(t, m_{z}^{2}, 0\right)}{s(s+u)}
\end{aligned}
$$

with $u=m_{H}^{2}-s-t$. Where we have expressed the form factor in terms of the 6-dimensional Box using the following relation

$$
\begin{aligned}
\operatorname{BoxE}^{(\mathrm{d}=6)}\left(s, t, m_{H}^{2}, m_{z}^{2}, 0\right)=-\frac{\left((s+t) m_{z}{ }^{2}-2 s t\right)\left((s+t) m_{z}{ }^{2}-s t\right)(-1+\epsilon) \operatorname{Tadp}\left(m_{z}{ }^{2}\right)}{2 \epsilon\left(m_{z}{ }^{2}-t\right) m_{z}{ }^{2}\left(m_{z}{ }^{2}-s\right)(-1+2 \epsilon) s t u} \\
+\frac{\left((s+t) m_{z}{ }^{2}-s t\right) \operatorname{BubE}\left(s, m_{z}{ }^{2}, 0\right)}{2 t u\left(m_{z}{ }^{2}-s\right) \epsilon}+\frac{\left((s+t) m_{z}{ }^{2}-s t\right) \operatorname{BubE}\left(t, m_{z}{ }^{2}, 0\right)}{2 s u\left(m_{z}{ }^{2}-t\right) \epsilon} \\
-\frac{\left(\left(s t+t^{2}-s u+t u\right) m_{z}{ }^{2}-s t(u+t)\right) \operatorname{TriaE}\left(s, m_{H}{ }^{2}, m_{z}{ }^{2}, 0\right)}{2(-1+2 \epsilon) s t u} \\
-\frac{\left(\left(s u+s^{2}-t u+s t\right) m_{z}^{2}-s t(s+u)\right) \operatorname{TriaE}\left(t, m_{h}{ }^{2}, m_{z}{ }^{2}, 0\right)}{2(-1+2 \epsilon) s t u} \\
+\frac{\left((s+t)^{2} m_{z}{ }^{4}-2 s t(s+t) m_{z}{ }^{2}+s^{2} t^{2}\right) \operatorname{BoxE}\left(s, t, m_{H}{ }^{2}, m_{z}{ }^{2}, 0\right)}{2(-1+2 \epsilon) s t u}
\end{aligned}
$$




\section{B.5 $A_{\mathrm{ewk}}^{m_{t}}$}

The form factor for the mixed QCD-electroweak corrections to $H+j$ with a top-quark in the loop is:

$$
\begin{aligned}
& A_{\mathrm{ewk}}^{m_{t}}\left(s, t, u, m_{w}^{2}, m_{t}^{2}\right)=\frac{m_{t}{ }^{2} \operatorname{Tadp}\left(m_{w}{ }^{2}\right)-m_{t}{ }^{2} \operatorname{Tadp}\left(m_{t}{ }^{2}\right)}{4 s^{2} m_{w}{ }^{2}} \\
& \frac{+m_{t}^{2}\left(-m_{w}^{2}+m_{t}^{2}\right) \operatorname{BubE}\left(s, m_{w}^{2}, m_{t}^{2}\right)}{4 s^{2} m_{w}{ }^{2}} \\
& +\frac{\left(-m_{H}^{2} m_{t}^{2}-2 m_{t}^{2} m_{w}{ }^{2}-4 m_{w}{ }^{4}\right) \operatorname{BubE}\left(t, m_{w}{ }^{2}, m_{t}{ }^{2}\right)}{4 s(u+s) m_{w}{ }^{4}} \\
& +\frac{\left(m_{H}{ }^{2} m_{t}{ }^{2}+2 m_{t}{ }^{2} m_{w}{ }^{2}+4 m_{w}{ }^{4}\right) \operatorname{Bub}\left(m_{H}{ }^{2}, m_{w}{ }^{2}\right)}{4 s(u+s) m_{w}{ }^{4}} \\
& +\left\{\left[-2 t(-t+s) m_{w}{ }^{2} m_{t}{ }^{6}-t(-t+s) m_{H}{ }^{2} m_{t}{ }^{6}-2 t(-t+s) m_{w}{ }^{4} m_{t}{ }^{4}\right.\right. \\
& +t\left(3 s^{2}+u s-t u-t^{2}\right) m_{w}{ }^{2} m_{t}{ }^{4}-t^{2} s m_{H}{ }^{2} m_{t}{ }^{4}+4 t(-t+s) m_{w}{ }^{6} m_{t}{ }^{2} \\
& \left.-2 t\left(s^{2}-s t\right) m_{t}{ }^{2} m_{w}{ }^{4}+2 s^{2} t^{2} m_{w}{ }^{2} m_{t}{ }^{2}\right] \operatorname{TriaF}\left(t, m_{w}{ }^{2}, m_{t}{ }^{2}\right) \\
& +\left[-(s+t) m_{H}{ }^{2} m_{t}{ }^{8}+(-2 t-2 s) m_{w}{ }^{2} m_{t}{ }^{8}-m_{H}{ }^{2}\left(t u+u s+t^{2}\right) m_{t}{ }^{6}\right. \\
& +\left(t^{2}+3 s^{2}+6 s t+t u+u s\right) m_{w}{ }^{2} m_{t}{ }^{6} \\
& +(2 s+2 t) m_{w}{ }^{4} m_{t}{ }^{6}+m_{H}{ }^{2}\left(s t u+t^{2} s\right) m_{t}{ }^{4} \\
& +\left(t u^{2}+t^{3}+3 s^{2} u+3 s t u+2 t^{2} s+2 t^{2} u-s^{2} t+s u^{2}\right) m_{w}{ }^{2} m_{t}{ }^{4} \\
& +\left(-5 t^{2}-7 s^{2}-5 u s-5 t u-8 s t\right) m_{w}{ }^{4} m_{t}{ }^{4}+(6 t+6 s) m_{w}{ }^{6} m_{t}{ }^{4} \\
& -2 s^{2} t(u+t) m_{w}{ }^{2} m_{t}{ }^{2}+\left(-3 s^{2} t-3 s t u-2 s^{2} u-3 t^{2} s\right) m_{w}{ }^{4} m_{t}{ }^{2} \\
& +\left(12 s t+5 t u+5 u s+9 s^{2}+5 t^{2}\right) m_{w}{ }^{6} m_{t}{ }^{2}+(-10 s-10 t) m_{w}{ }^{8} m_{t}{ }^{2}+4 m_{w}{ }^{6} s^{2} t \\
& \left.+\left(-8 s t-4 s^{2}\right) m_{w}{ }^{8}+(4 t+4 s) m_{w}{ }^{10}\right] \operatorname{TriaE}\left(s, m_{H}{ }^{2}, m_{w}{ }^{2}, m_{t}{ }^{2}\right) \\
& +\left[m_{t}{ }^{8}(s+t)^{2}+\left(-s^{2} u+3 t^{2} s+2 s^{2} t+5 s t u-s^{3}\right) m_{t}{ }^{6}\right. \\
& +\left(-4 s^{2}-2 t^{2}-2 s t\right) m_{w}{ }^{2} m_{t}^{6}-s^{2} t(u+s) m_{t}^{4} \\
& +\left(s^{2} u+3 s^{3}-s t u-2 s^{2} t-3 t^{2} s\right) m_{w}{ }^{2} m_{t}{ }^{4}+\left(-s^{2}+t^{2}+4 s t\right) m_{w}{ }^{4} m_{t}{ }^{4} \\
& \left.+2 s^{3} t m_{w}{ }^{2} m_{t}{ }^{2}-2 s^{2}(-t+s) m_{w}{ }^{4} m_{t}{ }^{2}+\left(-4 s t+4 s^{2}\right) m_{w}{ }^{6} m_{t}{ }^{2}\right] \operatorname{TriaF}\left(s, m_{w}{ }^{2}, m_{t}{ }^{2}\right) \\
& +\left[2 t(s+t) m_{w}{ }^{2} m_{t}{ }^{6}+t(s+t) m_{H}{ }^{2} m_{t}{ }^{6}\right. \\
& -2 t\left(s t+t u+t^{2}+s^{2}-u s\right) m_{t}^{4} m_{w}{ }^{2}+t m_{H}^{2}(s t+2 u s) m_{t}^{4} \\
& -6 t(s+t) m_{w}{ }^{6} m_{t}{ }^{2}+t\left(5 s^{2}+t^{2}+t u+9 u s+8 s t\right) m_{t}{ }^{2} m_{w}{ }^{4} \\
& -t\left(t^{2} s+4 s^{2} u+s^{2} t+s t u\right) m_{t}{ }^{2} m_{w}{ }^{2}+4 t(s+t) m_{w}{ }^{8}-4 t\left(2 s t+s^{2}\right) m_{w}{ }^{6} \\
& \left.\left.+4 m_{w}{ }^{4} s^{2} t^{2}\right] \operatorname{BoxE}^{(d=6)}\left(s, t, m_{H}{ }^{2}, m_{w}{ }^{2}, m_{t}{ }^{2}\right)\right\} \\
& \times\left[4 s m_{w}^{4}\left(4 m_{t}^{2} s t u+\left((s+t)\left(-m_{w}{ }^{2}+m_{t}^{2}\right)+s t\right)^{2}\right)\right]^{-1} \\
& +\left\{(s+t)(2 s+u+t) m_{h}{ }^{2} m_{t}{ }^{8}+2(s+t)(2 s+u+t) m_{w}{ }^{2} m_{t}{ }^{8}\right. \\
& -2(s+t)(2 s+u+t) m_{w}{ }^{4} m_{t}{ }^{6} \\
& +\left(-3 s t u-9 s^{2} t-4 s^{3}-s u^{2}-6 t^{2} s-3 t^{3}-4 t^{2} u-t u^{2}-5 s^{2} u\right) m_{w}{ }^{2} m_{t}{ }^{6}
\end{aligned}
$$




$$
\begin{aligned}
& +\left(t^{2} u+3 t^{2} s+6 s t u+t u^{2}+3 s^{2} t+s u^{2}+s^{3}+2 s^{2} u\right) m_{h}{ }^{2} m_{t}{ }^{6} \\
& -6(s+t)(2 s+u+t) m_{w}{ }^{6} m_{t}{ }^{4} \\
& +\left(17 s^{2} u+5 s u^{2}+27 s t u+5 t u^{2}+6 t^{2} u+17 s^{2} t+12 t^{2} s+12 s^{3}+3 t^{3}\right) m_{w}{ }^{4} m_{t}{ }^{4} \\
& +\left(-14 t^{2} s u-6 u^{2} s t-2 t^{3} u-9 s^{2} t^{2}-3 t^{2} u^{2}-t u^{3}-7 s^{3} u-5 s^{2} u^{2}-6 t^{3} s\right. \\
& \left.-s u^{3}-8 s^{3} t-3 s^{4}-13 s^{2} u t\right) m_{w}{ }^{2} m_{t}{ }^{4}+\left(2 s^{2} t^{2}+t^{2} s u+u^{2} s t+2 s^{2} u t+s^{3} t\right) m_{H}{ }^{2} m_{t}{ }^{4} \\
& +10(s+t)(2 s+u+t) m_{w}{ }^{8} m_{t}{ }^{2} \\
& +\left(-19 s^{2} u-5 s u^{2}-41 s t u-5 t u^{2}-8 t^{2} u-33 s^{2} t-22 t^{2} s-14 s^{3}-t^{3}\right) m_{w}{ }^{6} m_{t}{ }^{2} \\
& +\left(t^{3} u+t^{2} u^{2}+12 t^{2} s u+15 s^{2} t^{2}+12 u^{2} s t+2 s^{2} u^{2}+3 t^{3} s+4 s^{3} u\right. \\
& \left.+10 s^{3} t+2 s^{4}+22 s^{2} u t\right) m_{w}{ }^{4} m_{t}{ }^{2} \\
& -s t\left(2 s u^{2}+2 s^{3}+t^{2} u+t u^{2}+4 s^{2} u+3 s t u+2 t^{2} s+2 s^{2} t\right) m_{w}{ }^{2} m_{t}{ }^{2} \\
& -4(s+t)(2 s+u+t) m_{w}{ }^{10}+\left(4 t^{2} u+12 s t u+4 s^{2} u+20 s^{2} t+12 t^{2} s+4 s^{3}\right) m_{w}{ }^{8} \\
& \left.-4 s t m_{w}{ }^{6}\left(2 u s+3 s t+2 s^{2}+2 t u\right)+4 s^{2} t^{2}(u+s) m_{w}{ }^{2}\right\} \operatorname{TriaE}\left(t, m_{H}{ }^{2}, m_{w}{ }^{2}, m_{t}{ }^{2}\right) \\
& \times\left[4 s(u+s) m_{w}{ }^{4}\left(4 m_{t}{ }^{2} s t u+\left((s+t)\left(-m_{w}{ }^{2}+m_{t}{ }^{2}\right)+s t\right)^{2}\right)\right]^{-1}
\end{aligned}
$$

where

$$
\begin{aligned}
\operatorname{BoxE}^{(d=6)}\left(s, t, m_{H}{ }^{2}, m_{w}{ }^{2}, m_{t}{ }^{2}\right)=\left(s^{2} t^{2}-2(s+t)^{2} m_{t}{ }^{2} m_{w}{ }^{2}-2 s t(s+t) m_{w}{ }^{2}\right. \\
\left.+2 s t(t+s+2 u) m_{t}{ }^{2}+(s+t)^{2} m_{w}{ }^{4}+(s+t)^{2} m_{t}{ }^{4}\right) \frac{\operatorname{BoxE}\left(s, t, m_{H}{ }^{2}, m_{w}{ }^{2}, m_{t}^{2}\right)}{2 s t u(-1+2 \epsilon)} \\
+\frac{\left(\left(s u-t u-s t-t^{2}\right) m_{w}{ }^{2}+\left(t u+s t-s u+t^{2}\right) m_{t}{ }^{2}+s t(u+t)\right) \operatorname{TriaE}\left(s, m_{H}{ }^{2}, m_{w}{ }^{2}, m_{t}{ }^{2}\right)}{2 s t u(-1+2 \epsilon)} \\
+\frac{\left(\left(-s t-s u+t u-s^{2}\right) m_{w}{ }^{2}+\left(s u+s t-t u+s^{2}\right) m_{t}{ }^{2}+s t(s+u)\right) \operatorname{TriaE}\left(t, m_{H}{ }^{2}, m_{w}{ }^{2}, m_{t}{ }^{2}\right)}{2 s t u(-1+2 \epsilon)} \\
-\frac{\left(s \operatorname{TriaF}\left(s, m_{w}{ }^{2}, m_{t}{ }^{2}\right)+t \operatorname{TriaF}\left(t, m_{w}{ }^{2}, m_{t}{ }^{2}\right)\right)\left((-t-s) m_{w}{ }^{2}+(s+t) m_{t}{ }^{2}+s t\right)}{2 s t u(-1+2 \epsilon)}
\end{aligned}
$$

and $m_{H}^{2}=s+t+u$. The $m_{t} \rightarrow 0$ limit of this form factor trivially lead to $A_{\text {ewk }}$ of eq. (B.7).

\section{References}

[1] LEP Working Group for Higgs boson searches and AlEPH, DELPHI L3, OPAL collaborations, R. Barate et al., Search for the standard model Higgs boson at LEP, Phys. Lett. B 565 (2003) 61 [hep-ex/0306033] [INSPIRE].

[2] CDF and D0 collaborations, T. Aaltonen et al., Combined CDF and D0 upper limits on standard model Higgs boson production with up to 8.2 $\mathrm{fb}^{-1}$ of data, arXiv:1103.3233 [INSPIRE].

[3] ATLAS collaboration, G. Aad et al., Limits on the production of the standard model Higgs boson in pp collisions at $\sqrt{s}=7 \mathrm{TeV}$ with the ATLAS detector, Eur. Phys. J. C 71 (2011) 1728 [arXiv:1106.2748] [INSPIRE].

[4] CMS collaboration, S. Chatrchyan et al., Measurement of $W^{+} W^{-}$production and search for the Higgs boson in pp collisions at $\sqrt{s}=7$ TeV, Phys. Lett. B 699 (2011) 25 [arXiv:1102.5429] [INSPIRE]. 
[5] W.J. Marciano, Constraints and speculations on fourth generation physics, Annals N. Y. Acad. Sci. 518 (1987) 180 [InSPIRE].

[6] C.T. Hill, Theoretical expectations for mass scales of the fourth generation and Higgs bosons, Annals N. Y. Acad. Sci. 518 (1987) 168 [INSPIRE].

[7] E.W.N. Glover, J. Ohnemus and S.S.D. Willenbrock, The intermediate mass Higgs boson and the fourth generation, Phys. Lett. B 206 (1988) 696 [INSPIRE].

[8] V.D. Barger et al., Higgs boson Z0 associated production from fourth generation quarks at super collider energies, Phys. Rev. Lett. 57 (1986) 1672 [INSPIRE].

[9] A. Falkowski, Pseudo-goldstone Higgs production via gluon fusion, Phys. Rev. D 77 (2008) 055018 [arXiv:0711.0828] [inSPIRE].

[10] G.F. Giudice, C. Grojean, A. Pomarol and R. Rattazzi, The strongly-interacting light Higgs, JHEP 06 (2007) 045 [hep-ph/0703164] [INSPIRE].

[11] E. Furlan, Gluon-fusion Higgs production at NNLO for a non-standard Higgs sector, JHEP 10 (2011) 115 [arXiv:1106.4024] [INSPIRE].

[12] T.P. Cheng and M. Sher, Mass matrix ansatz and flavor nonconservation in models with multiple Higgs doublets, Phys. Rev. D 35 (1987) 3484 [INSPIRE].

[13] K.S. Babu and S. Nandi, Natural fermion mass hierarchy and new signals for the Higgs boson, Phys. Rev. D 62 (2000) 033002 [hep-ph/9907213] [INSPIRE].

[14] G.F. Giudice and O. Lebedev, Higgs-dependent Yukawa couplings, Phys. Lett. B 665 (2008) 79 [arXiv:0804.1753] [INSPIRE].

[15] J.J. van der Bij and S. Dilcher, HEIDI and the unparticle, Phys. Lett. B 655 (2007) 183 [arXiv:0707.1817] [INSPIRE].

[16] J.J. van der Bij and B. Pulice, New spectra in the HEIDI Higgs models, Nucl. Phys. B 853 (2011) 49 [arXiv: 1104.2062] [InSPIRE].

[17] H. Georgi, Unparticle physics, Phys. Rev. Lett. 98 (2007) 221601 [hep-ph/0703260] [INSPIRE].

[18] S. Buehler and C. Duhr, CHAPLIN - complex harmonic polylogarithms in Fortran, arXiv:1106.5739 [INSPIRE].

[19] A. van Hameren, OneLOop: for the evaluation of one-loop scalar functions, Comput. Phys. Commun. 182 (2011) 2427 [arXiv: 1007.4716] [inSPIRE].

[20] A. van Hameren, C.G. Papadopoulos and R. Pittau, Automated one-loop calculations: a proof of concept, JHEP 09 (2009) 106 [arXiv: 0903.4665] [INSPIRE].

[21] R.K. Ellis and G. Zanderighi, Scalar one-loop integrals for QCD, JHEP 02 (2008) 002 [arXiv:0712.1851] [INSPIRE].

[22] http://hepforge.cedar.ac.uk/lhapdf/.

[23] S. Alekhin, J. Blumlein, S. Klein and S. Moch, The 3, 4 and 5-flavor NNLO parton from deep-inelastic-scattering data and at hadron colliders, Phys. Rev. D 81 (2010) 014032 [arXiv:0908.2766] [INSPIRE].

[24] A.D. Martin, W.J. Stirling, R.S. Thorne and G. Watt, Parton distributions for the LHC, Eur. Phys. J. C 63 (2009) 189 [arXiv:0901.0002] [INSPIRE]. 
[25] P. Jimenez-Delgado and E. Reya, Variable flavor number parton distributions and weak gauge and Higgs boson production at hadron colliders at NNLO of QCD,

Phys. Rev. D 80 (2009) 114011 [arXiv:0909.1711] [INSPIRE].

[26] A. Djouadi, J. Kalinowski and M. Spira, HDECAY: a program for Higgs boson decays in the standard model and its supersymmetric extension, Comput. Phys. Commun. 108 (1998) 56 [hep-ph/9704448] [INSPIRE].

[27] D. Graudenz, M. Spira and P.M. Zerwas, QCD corrections to Higgs boson production at proton proton colliders, Phys. Rev. Lett. 70 (1993) 1372 [INSPIRE].

[28] M. Spira, A. Djouadi, D. Graudenz and P.M. Zerwas, Higgs boson production at the LHC, Nucl. Phys. B 453 (1995) 17 [hep-ph/9504378] [INSPIRE].

[29] S. Dawson, Radiative corrections to Higgs boson production, Nucl. Phys. B 359 (1991) 283 [INSPIRE].

[30] A. Djouadi, M. Spira and P.M. Zerwas, Production of Higgs bosons in proton colliders: QCD corrections, Phys. Lett. B 264 (1991) 440 [inSPIRE].

[31] R. Harlander and P. Kant, Higgs production and decay: analytic results at next-to-leading order QCD, JHEP 12 (2005) 015 [hep-ph/0509189] [INSPIRE].

[32] C. Anastasiou, S. Beerli, S. Bucherer, A. Daleo and Z. Kunszt, Two-loop amplitudes and master integrals for the production of a Higgs boson via a massive quark and a scalar-quark loop, JHEP 01 (2007) 082 [hep-ph/0611236] [INSPIRE].

[33] U. Aglietti, R. Bonciani, G. Degrassi and A. Vicini, Analytic results for virtual QCD corrections to Higgs production and decay, JHEP 01 (2007) 021 [hep-ph/0611266] [INSPIRE].

[34] R.K. Ellis, I. Hinchliffe, M. Soldate and J.J. van der Bij, Higgs decay to $\tau^{+} \tau^{-}$: a possible signature of intermediate mass Higgs bosons at the SSC, Nucl. Phys. B 297 (1988) 221 [INSPIRE].

[35] U. Baur and E.W.N. Glover, Higgs boson production at large transverse momentum in hadronic collisions, Nucl. Phys. B 339 (1990) 38 [INSPIRE].

[36] R. Bonciani, G. Degrassi and A. Vicini, Scalar particle contribution to Higgs production via gluon fusion at NLO, JHEP 11 (2007) 095 [arXiv:0709.4227] [INSPIRE].

[37] C. Anastasiou, S. Bucherer and Z. Kunszt, HPro: a NLO Monte-Carlo for Higgs production via gluon fusion with finite heavy quark masses, JHEP 10 (2009) 068 [arXiv:0907.2362] [INSPIRE].

[38] C. Anastasiou, R. Boughezal and E. Furlan, The NNLO gluon fusion Higgs production cross-section with many heavy quarks, JHEP 06 (2010) 101 [arXiv:1003.4677] [INSPIRE].

[39] K.G. Chetyrkin, B.A. Kniehl and M. Steinhauser, Hadronic Higgs decay to order $\alpha_{s}^{4}$, Phys. Rev. Lett. 79 (1997) 353 [hep-ph/9705240] [INSPIRE].

[40] M. Krämer, E. Laenen and M. Spira, Soft gluon radiation in Higgs boson production at the LHC, Nucl. Phys. B 511 (1998) 523 [hep-ph/9611272] [INSPIRE].

[41] R.V. Harlander and W.B. Kilgore, Soft and virtual corrections to proton proton $\rightarrow H+x$ at NNLO, Phys. Rev. D 64 (2001) 013015 [hep-ph/0102241] [INSPIRE].

[42] S. Catani, D. de Florian and M. Grazzini, Higgs production in hadron collisions: soft and virtual QCD corrections at NNLO, JHEP 05 (2001) 025 [hep-ph/0102227] [INSPIRE]. 
[43] R.V. Harlander and W.B. Kilgore, Next-to-next-to-leading order Higgs production at hadron colliders, Phys. Rev. Lett. 88 (2002) 201801 [hep-ph/0201206] [INSPIRE].

[44] C. Anastasiou and K. Melnikov, Higgs boson production at hadron colliders in NNLO QCD, Nucl. Phys. B 646 (2002) 220 [hep-ph/0207004] [INSPIRE].

[45] V. Ravindran, J. Smith and W.L. van Neerven, NNLO corrections to the total cross-section for Higgs boson production in hadron hadron collisions, Nucl. Phys. B 665 (2003) 325 [hep-ph/0302135] [INSPIRE].

[46] S. Actis, G. Passarino, C. Sturm and S. Uccirati, NNLO computational techniques: the cases $H \rightarrow \gamma \gamma$ and $H \rightarrow g g$, Nucl. Phys. B 811 (2009) 182 [arXiv:0809.3667] [InSPIRE].

[47] S. Actis, G. Passarino, C. Sturm and S. Uccirati, NLO electroweak corrections to Higgs boson production at hadron colliders, Phys. Lett. B 670 (2008) 12 [arXiv:0809.1301] [INSPIRE].

[48] U. Aglietti, R. Bonciani, G. Degrassi and A. Vicini, Two loop light fermion contribution to Higgs production and decays, Phys. Lett. B 595 (2004) 432 [hep-ph/0404071] [INSPIRE].

[49] W.-Y. Keung and F.J. Petriello, Electroweak and finite quark-mass effects on the Higgs boson transverse momentum distribution, Phys. Rev. D 80 (2009) 013007 [arXiv:0905.2775] [INSPIRE].

[50] C. Anastasiou, R. Boughezal and F. Petriello, Mixed QCD-electroweak corrections to Higgs boson production in gluon fusion, JHEP 04 (2009) 003 [arXiv: 0811.3458] [INSPIRE].

[51] J.M. Campbell et al., Higgs boson production in association with bottom quarks, hep-ph/0405302 [INSPIRE].

[52] S. Dittmaier, M. Krämer and M. Spira, Higgs radiation off bottom quarks at the Tevatron and the CERN LHC, Phys. Rev. D 70 (2004) 074010 [hep-ph/0309204] [INSPIRE].

[53] S. Dawson, C.B. Jackson, L. Reina and D. Wackeroth, Exclusive Higgs boson production with bottom quarks at hadron colliders, Phys. Rev. D 69 (2004) 074027 [hep-ph/0311067] [INSPIRE].

[54] D. Dicus, T. Stelzer, Z. Sullivan and S. Willenbrock, Higgs boson production in association with bottom quarks at next-to-leading order, Phys. Rev. D 59 (1999) 094016 [hep-ph/9811492] [INSPIRE].

[55] E. Boos and T. Plehn, Higgs boson production induced by bottom quarks, Phys. Rev. D 69 (2004) 094005 [hep-ph/0304034] [INSPIRE].

[56] F. Maltoni, Z. Sullivan and S. Willenbrock, Higgs-boson production via bottom-quark fusion, Phys. Rev. D 67 (2003) 093005 [hep-ph/0301033] [INSPIRE].

[57] R.V. Harlander and W.B. Kilgore, Higgs boson production in bottom quark fusion at next-to-next-to leading order, Phys. Rev. D 68 (2003) 013001 [hep-ph/0304035] [InSPIRE].

[58] http://particle.uni-wuppertal.de/harlander/software/bbh@nnlo/.

[59] M. Beneke, A.P. Chapovsky, A. Signer and G. Zanderighi, Effective theory calculation of resonant high-energy scattering, Nucl. Phys. B 686 (2004) 205 [hep-ph/0401002] [INSPIRE].

[60] A. Denner, S. Dittmaier, M. Roth and L.H. Wieders, Electroweak corrections to charged-current $e^{+} e^{-} \rightarrow 4$ fermion processes: technical details and further results, Nucl. Phys. B 724 (2005) 247 [hep-ph/0505042] [INSPIRE].

[61] G. Zanderighi, Effective theory approach to unstable particles, hep-ph/0405124 [INSPIRE]. 
[62] E.W.N. Glover and J.J. van der Bij, Z boson pair production via gluon fusion, Nucl. Phys. B 321 (1989) 561 [inSPIRE].

[63] E.W.N. Glover and J.J. van der Bij, Vector boson pair production via gluon fusion, Phys. Lett. B 219 (1989) 488 [inSPIRE].

[64] U. Baur and E.W.N. Glover, $Z$ boson pair production via vector boson scattering and the search for the Higgs boson at hadron supercolliders, Nucl. Phys. B 347 (1990) 12 [InSPIRE].

[65] G. Valencia and S. Willenbrock, The heavy Higgs resonance, Phys. Rev. D 46 (1992) 2247 [INSPIRE].

[66] M.H. Seymour, The Higgs boson line shape and perturbative unitarity, Phys. Lett. B 354 (1995) 409 [hep-ph/9505211] [InSPIRE].

[67] G. Passarino, C. Sturm and S. Uccirati, Higgs pseudo-observables, second Riemann sheet and all that, Nucl. Phys. B 834 (2010) 77 [arXiv:1001.3360] [inSPIRE].

[68] S. Alekhin, J. Blumlein, P. Jimenez-Delgado, S. Moch and E. Reya, NNLO benchmarks for gauge and Higgs boson production at TeV hadron colliders, Phys. Lett. B 697 (2011) 127 [arXiv: 1011.6259] [INSPIRE].

[69] C. Anastasiou, S. Buehler, E. Furlan, F. Herzog and A. Lazopoulos, Higgs production cross-section in a standard model with four generations at the LHC, Phys. Lett. B 702 (2011) 224 [arXiv:1103.3645] [INSPIRE].

[70] R.V. Harlander and K.J. Ozeren, Finite top mass effects for hadronic Higgs production at next-to-next-to-leading order, JHEP 11 (2009) 088 [arXiv:0909.3420] [INSPIRE].

[71] A. Pak, M. Rogal and M. Steinhauser, Finite top quark mass effects in NNLO Higgs boson production at LHC, JHEP 02 (2010) 025 [arXiv:0911.4662] [INSPIRE].

[72] ATLAS collaboration, G. Aad et al., Expected performance of the ATLAS experiment: detector, trigger and physics, CERN-OPEN-2008-020 [arXiv:0901.0512] [INSPIRE].

[73] D. de Florian and M. Grazzini, Higgs production through gluon fusion: updated cross sections at the Tevatron and the LHC, Phys. Lett. B 674 (2009) 291 [arXiv:0901.2427] [INSPIRE].

[74] V. Ahrens, T. Becher, M. Neubert and L.L. Yang, Updated predictions for Higgs production at the Tevatron and the LHC, Phys. Lett. B 698 (2011) 271 [arXiv:1008.3162] [INSPIRE].

[75] J. Baglio and A. Djouadi, Higgs production at the LHC, JHEP 03 (2011) 055 [arXiv: 1012.0530] [INSPIRE].

[76] S. Moch and A. Vogt, Higher-order soft corrections to lepton pair and Higgs boson production, Phys. Lett. B 631 (2005) 48 [hep-ph/0508265] [INSPIRE].

[77] S. Alioli, P. Nason, C. Oleari and E. Re, NLO Higgs boson production via gluon fusion matched with shower in POWHEG, JHEP 04 (2009) 002 [arXiv:0812.0578] [INSPIRE].

[78] T. Hahn, CUBA: a library for multidimensional numerical integration, Comput. Phys. Commun. 168 (2005) 78 [hep-ph/0404043] [INSPIRE].

[79] M.R. Whalley, D. Bourilkov and R.C. Group, The Les Houches accord PDFs (LHAPDF) and LHAGLUE, hep-ph/0508110 [INSPIRE].

[80] O. Brein, Electroweak and bottom quark contributions to Higgs boson plus jet production, Phys. Rev. D 81 (2010) 093006 [arXiv: 1003.4438] [INSPIRE]. 